\title{
LIMITS OF POSITIVE FLAT BIVARIATE MOMENT MATRICES
}

\author{
LAWRENCE A. FIALKOW
}

\begin{abstract}
The bivariate moment problem for a sequence $\beta \equiv \beta^{(6)}$ of degree 6 remains unsolved, but we prove that if the associated $10 \times 10$ moment matrix $M_{3}(\beta)$ satisfies $M_{3} \succeq 0$ and $\operatorname{rank} M_{3} \leq 6$, then $\beta$ admits a sequence of approximate representing measures, and $\beta^{(5)}$ has a representing measure. More generally, let $\overline{\mathcal{F}_{d}}$ denote the closure of the positive flat moment matrices of degree $2 d$ in $n$ variables. Each matrix in $\overline{\mathcal{F}_{d}}$ admits computable approximate representing measures, and in 2013, Jiawang Nie and the author began to study concrete conditions for membership in this class. Let $\beta \equiv \beta^{(2 d)}=\left\{\beta_{i}\right\}_{i \in \mathbb{Z}_{+}^{n},|i| \leq 2 d}$, $\beta_{0}>0$, denote a real $n$-dimensional sequence of degree $2 d$. If the corresponding moment matrix $M_{d} \equiv M_{d}(\beta)$ is the limit of a sequence of positive flat moment matrices $\left\{M_{d}^{(k)}\right\}$, i.e., $M_{d}^{(k)} \succeq 0$ and $\operatorname{rank} M_{d}^{(k)}=\operatorname{rank} M_{d-1}^{(k)}$, then i) $M_{d} \succeq 0$, ii) $\operatorname{rank} M_{d} \leq \rho_{d-1} \equiv \operatorname{dim} \mathbb{R}\left[x_{1}, \ldots, x_{n}\right]_{d-1}$, and iii) $\beta^{(2 d-1)}$ admits a representing measure. We extend our earlier results by proving, conversely, that for $n=2$, if $M_{d}$ satisfies certain positivity and rank conditions related to i)-iii), then $M_{d}$ is the limit of positive flat moment matrices.
\end{abstract}

\section{INTRODUCTION}

Let $\beta \equiv \beta^{(m)}=\left\{\beta_{i}\right\}_{i \in \mathbb{Z}_{+}^{n},|i| \leq m}$ denote a real $n$-dimensional sequence of degree $m, \beta_{0}>0$, and let $K \subseteq \mathbb{R}^{n}$ be a closed set. The Truncated $K$-Moment Problem (TKMP) concerns conditions for the existence of a positive Borel measure $\mu$, supported in $K$, such that

$$
\beta_{i}=\int x^{i} d \mu(|i| \leq m)
$$

(where $i \equiv\left(i_{1}, \ldots, i_{n}\right) \in \mathbb{Z}_{+}^{n},|i|=i_{1}+\cdots+i_{n}, x \equiv\left(x_{1}, \ldots, x_{n}\right) \in \mathbb{R}^{n}$, and $\left.x^{i}:=x_{1}^{i_{1}} \cdots x_{n}^{i_{n}}\right) . \quad \beta$ as above is a truncated moment sequence, and we refer to $\mu$ as a $K$-representing measure for $\beta$. In the case $K=\mathbb{R}^{n}$, we refer to TKMP as the Truncated Moment Problem (TMP) and to $\mu$ as a representing measure. Although several abstract criteria for the existence of representing measures are known [CF9], CF7] (cf. Theorems 1.2 and 1.4 below), the only concrete condition available is flatness of the moment data, i.e., the moment matrix $M_{d}$ associated to $\beta^{(2 d)}$ satisfies $M_{d} \succeq 0$ (positive semidefinite) and $\operatorname{rank} M_{d}=\operatorname{rank} M_{d-1}$ [CF7] (cf. Theorem 1.4). With the aid of this condition, TKMP has been solved concretely for $K$ a planar curve of degree 1 or 2 [CF3], CF5], CF6], CF8], and for $y=x^{3}$ $[\mathrm{F}$. In particular, for $n=2$, TMP has been solved concretely for degree $2(d=1)$ and degree $4(d=2)$ CF3, CF5]. Nevertheless, the degree 6 problem $(d=3)$

Received by the editors February 10, 2013.

2010 Mathematics Subject Classification. Primary 47A57, 44A60, 42A70, 30E05; Secondary 15A57, 15-04, 47A20.

Key words and phrases. Truncated moment problem, Riesz functional, moment matrix extension, flat extensions of positive matrices, positive functional. 
remains largely unsolved, and similarly for $\beta^{(m)}$ with $m \geq 5$. In view of this difficulty, our focus here is on membership of $M_{d}$ in $\overline{\mathcal{F}_{d}}$, the closure of the positive flat moment matrices. For if $M_{d}(\beta)$ belongs to $\overline{\mathcal{F}_{d}}$, then the moment problem for $\beta$ is "almost" solved in two respects: first, there exists a sequence of approximate representing measures for $\beta$ and, second, as described by Theorem 1.2 (cf. CF9]), $\beta^{(2 d-1)}$ has a representing measure. Moreover, as we discuss below, the existence of approximate representing measures provides a criterion for finite convergence in the polynomial optimization program of J.-B. Lasserre [Las]. Our main result, Theorem [1.9, provides a sufficient condition for membership in $\overline{\mathcal{F}_{d}}$. The main application of Theorem 1.9 is the following simple criterion for approximation by positive flat moment matrices in the degree 6 problem.

Theorem 1.1. Let $n=2$ and let $\beta \equiv \beta^{(6)}=\left\{\beta_{i j}\right\}_{i, j \geq 0, i+j \leq 6}$. The associated $10 \times 10$ moment matrix $M_{3} \equiv M_{3}(\beta)$ belongs to $\overline{\mathcal{F}_{3}}$ if and only if $M_{3} \succeq 0$ and $\rho \equiv$ rank $M_{3} \leq 6$. In this case, given $\tau, \rho \leq \tau \leq 6, M_{3}$ is in the closure of the rank- $\tau$ positive flat moment matrices, and there exists a sequence of (computable) $\tau$ atomic positive measures $\left\{\mu_{k}\right\}$ such that $\beta_{i j}=\lim _{k \rightarrow \infty} \int x^{i} y^{j} d \mu_{k}(i, j \geq 0, i+j \leq 6)$. Moreover, $\beta^{(5)}$ has a representing measure.

Let $\mathbb{R}[x]_{m} \equiv \mathbb{R}\left[x_{1}, \ldots, x_{n}\right]_{m}:=\left\{p \in \mathbb{R}\left[x_{1}, \ldots, x_{n}\right]:\right.$ deg $\left.p \leq m\right\}$. We associate to $\beta$ the Riesz functional $L_{\beta}: \mathbb{R}[x]_{m} \mapsto \mathbb{R}$ defined by $L_{\beta}\left(\sum_{|i| \leq m} a_{i} x^{i}\right)=\sum a_{i} \beta_{i}$. If

$\beta$ has a $K$-representing measure $\mu$, then $L_{\beta}$ is $K$-positive, i.e., $p \in \mathbb{R}[x]_{m}, p \mid K \geq$ $0 \Longrightarrow L_{\beta}(p) \geq 0$; indeed, in this case, $L_{\beta}(p)=\int_{K} p d \mu \geq 0$. For $K=\mathbb{R}^{n}$, we say that $L_{\beta}$ is positive. In the classical Full Multidimensional $K$-Moment Problem for $\beta \equiv \beta^{(\infty)}$, the Riesz-Haviland Theorem $[\mathrm{R}],[\mathrm{H}]$ states that $\beta$ admits a $K$ representing measure if and only if $L_{\beta}$ is $K$-positive. Such is not the case in TKMP. The proof of Tchakaloff's Theorem [T] shows that if $K$ is compact, then $K$-positivity of $L_{\beta}$ does imply that $\beta$ has a (finitely atomic) $K$-representing measure. However, for $K$ noncompact, this implication fails; for example, with $n=1, K=\mathbb{R}, m=4$, the sequence 1, 1, 1, 1, 2 has a positive functional but no representing measure (cf. Example 1.5). The following result of [CF9] illustrates the role of $K$-positivity in TKMP.

Theorem 1.2 ([CF9, Theorem 1.2]). $\beta \equiv \beta^{(2 d)}$ (or $\left.\beta \equiv \beta^{(2 d+1)}\right)$ admits a $K$ representing measure if and only if $\beta$ can be extended to a sequence $\widetilde{\beta} \equiv \widetilde{\beta}^{(2 d+2)}$ for which $L_{\widetilde{\beta}}$ is $K$-positive.

(The result in CF9 is stated only for $\beta^{(2 d)}$, but it is clear from the proof that it applies as well to $\beta^{(2 d+1)}$.)

$K$-positivity also solves TKMP in the sense of approximation. Let $\mathcal{M}_{n, m}$ denote the set of all real $n$-dimensional sequences of degree $m$, viewed as a subset of $\mathbb{R}^{\eta}$ (endowed with the Euclidean norm), where $\eta:=\operatorname{dim} \mathbb{R}[x]_{m}\left(=\left(\begin{array}{c}n+m \\ m\end{array}\right)\right)$. Let $\mathcal{R}_{n, m}(K):=\left\{\beta \in \mathcal{M}_{n, m}: \beta\right.$ has a K-representing measure $\}$, and let $\mathcal{P}_{n, m}^{m}(K):=$ $\left\{\beta \in \mathcal{M}_{n, m}: L_{\beta}\right.$ is $K$-positive $\}$. Note that $\mathcal{R}_{n, m}(K) \subset \mathcal{P}_{n, m}(K) \subset \mathcal{M}_{n, m}$ is an inclusion of convex cones, with $\mathcal{M}_{n, m}$ and $\mathcal{P}_{n, m}(K)$ closed; it will become clear in the sequel that in general $\mathcal{R}_{n, m}(K)$ is not closed.

Theorem 1.3 ([FN1, Theorem 2.2]). $\mathcal{P}_{n, m}(K)=\overline{\mathcal{R}_{n, m}(K)}$. If $L_{\beta^{(m)}}$ is $K$-positive, then there exists a sequence of positive Borel measures $\left\{\mu_{k}\right\}$, each supported in 
$K$, having finite moments up to degree $m$, such that for each $i,|i| \leq m, \beta_{i}=$ $\lim _{k \rightarrow \infty} \int x^{i} d \mu_{k}$.

In view of the preceding two results, it would be desirable to have a concrete test for $K$-positivity, but at present there is no such test that is applicable to a general multisequence. Similarly, in the Full $K$-Moment Problem, concrete conditions for $K$-positivity are known only in certain cases, e.g., in Hamburger's solution for $K=\mathbb{R}$ (cf. $[\mathrm{A},[\mathrm{KN}]$ ) and in $\mathrm{K}$. Schmüdgen's solution for $K$ a basic compact semialgebraic set $[\mathrm{S} 2$. Remarkably, in the compact case treated by Tchakaloff [S2], there is no known concrete test for $K$-positivity in TKMP, even in the case when $K$ is a basic compact semialgebraic set. In FN2] we began to study conditions for $\beta$ to be a limit of "flat" multisequences (as defined below), in which case positivity of $L_{\beta}$ is obvious. Moreover, if the flat approximants $\beta^{[k]}$ are known, then corresponding approximate representing measures $\mu^{(k)}$ for $\beta$ can be explicitly computed (cf. Example 1.5). In FN2 we obtained concrete necessary and sufficient conditions for flat approximation when $n=1, d \geq 1$ and when $n=d=2$ (cf. Theorem 1.6 below). In the present note, we extend the results of [FN2] to the bivariate truncated moment problem, i.e., $n=2, d \geq 1$. The conditions that we present in Theorem 1.9 apply in certain cases in which the truncated moment problem is still unsolved, including certain cases of the bivariate degree 6 problem, so we can at least test for approximate representing measures in these cases; Theorem 1.1 provides one such test.

To describe our results in detail, we require some additional terminology. Following [CF7], we associate to $\beta \equiv \beta^{(2 d)}$ the moment matrix $M_{d}(\beta)$. For $p \in \mathbb{R}[x]_{d}$, $p=\sum_{|i| \leq d} a_{i} x^{i}$, let $\widehat{p} \equiv\left(a_{i}\right)$ denote the vector of coefficients of $p$ with respect to the basis for $\mathbb{R}[x]_{d}$ consisting of the monomials in degree-lexicographic order. Let $\rho_{d}=\operatorname{dim} \mathbb{R}[x]_{d}$. Then the moment matrix $M_{d} \equiv M_{d}(\beta)$ is the $\rho_{d} \times \rho_{d}$ matrix defined by

$$
\left\langle M_{d}(\beta) \widehat{p}, \widehat{q}\right\rangle=L_{\beta}(p q)\left(p, q \in \mathbb{R}[x]_{d}\right) .
$$

As noted above, if $\beta$ has a representing measure, then $L_{\beta}$ is positive. Further, if $L_{\beta}$ is positive, then $M_{d}(\beta)$ is positive semidefinite $\left(M_{d}(\beta) \succeq 0\right)$, since $\left\langle M_{d}(\beta) \widehat{p}, \widehat{p}\right\rangle=$ $L_{\beta}\left(p^{2}\right) \geq 0\left(p \in \mathbb{R}[x]_{d}\right)$. In general, the preceding implication cannot be reversed, but there is one situation where positivity of $M_{d}(\beta)$ is equivalent to positivity of $L_{\beta}$. Recall that $p \in \mathbb{R}[x]_{2 d}$ is nonnegative or positive semidefinite (psd) if $p \mid \mathbb{R}^{n} \geq$ 0 , and $p$ is a sum of squares (SOS) if there exist $p_{1}, \ldots, p_{k} \in \mathbb{R}[x]_{d}$ such that $p=\sum_{j=1}^{k} p_{j}^{2}$. Now, if every nonnegative polynomial $p$ in $\mathbb{R}[x]_{2 d}$ is SOS , then positive semidefiniteness of $M_{d}(\beta)$ implies positivity of $L_{\beta}$, since, for $p$ nonnegative, $L_{\beta}(p)=$ $L_{\beta}\left(\sum_{j=1}^{k} p_{j}^{2}\right)=\sum\left\langle M_{d}(\beta) \widehat{p}_{j}, \widehat{p}_{j}\right\rangle \geq 0$. A well-known theorem of Hilbert (cf. [Rez]) shows that each psd polynomial is SOS if and only if $n=1, d \geq 1 ; n=d=2$; or $n \geq 1, d=1$. In each of these cases, checking that $L_{\beta}$ is positive reduces to simply verifying that $M_{d}(\beta)$ is positive semidefinite. Now, for $n=2, d=3$, there exists $M_{3}(\beta) \succ 0$ (positive definite) for which $L_{\beta}$ is not positive CF3, [S1]; more generally, [FN2, Proposition 1.6] shows that except in the cases of Hilbert's 
theorem, positive semidefiniteness of a moment matrix does not imply positivity of its Riesz functional.

In the sequel we seek to identify cases of Riesz functional positivity that are beyond the scope of Hilbert's theorem and are not due to the existence of representing measures. The first such examples appear in $\mathrm{EF}$ for certain bivariate $M_{3}$ with rank $M_{3}=9$. In the present note we focus on positivity arising from approximation by positive flat moment matrices. The following result of [CF7 illustrates the central role of positive flat moment matrices in TMP.

Theorem 1.4 ([CF7]). Let $\beta \equiv \beta^{(2 d)}$. If $M_{d}(\beta) \succeq 0$ is flat, i.e., rank $M_{d}=$ rank $M_{d-1}$, then $\beta$ admits a unique representing measure which is rank $M_{d}(\beta)$ atomic. More generally, $\beta$ has a representing measure if and only if $\beta$ can be extended to a sequence $\widetilde{\beta} \equiv \widetilde{\beta}^{(2(d+k))}$ (for some $\left.k, 0 \leq k \leq \rho_{2 d}-\operatorname{rank} M_{d}(\beta)+1\right)$ for which $M_{d+k}(\widetilde{\beta})$ is positive semidefinite and flat.

The result in [CF7] is stated in terms of finitely atomic representing measures, but in [BT] Bayer and Teichmann proved that the existence of a representing measure implies the existence of a finitely atomic representing measure. Theorem 1.4 is the basis for a concrete solution to TKMP when $K$ is the real line $\mathbb{R}$ CF1, a planar line or curve of degree 2 [CF3, CF5], CF6], CF8], or the planar curve $y=x^{3}$ $[\mathrm{F}$. Theorem 1.4 also leads to an algorithmic solution to TMP for the class of recursively determinate moment matrices CF10 and is used by Helton and Nie [HN] in a numerical solution to TKMP based on semidefinite programming (see also [FN3]).

Let $\mathcal{F}_{d}:=\left\{M_{d}(\beta): M_{d}(\beta) \succeq 0, \operatorname{rank} M_{d}(\beta)=\operatorname{rank} M_{d-1}(\beta)\right\}$, the set of positive flat moment matrices with data of degree $2 d$. For $M_{d}(\beta) \in \mathcal{F}_{d}$, we refer to $\beta$ as a flat multisequence. Now if $M_{d}(\beta)$ belongs to $\overline{\mathcal{F}_{d}}$, the closure of $\mathcal{F}_{d}$ in any of the (equivalent) norms on the $\rho_{d} \times \rho_{d}$ matrices, then, by Theorems 1.3 and 1.4, $L_{\beta}$ is positive. Moreover, if $M_{d}(\beta)=\lim _{k \rightarrow \infty} M_{d}^{(k)}$, where each $M_{d}^{(k)}$ is positive semidefinite and flat, then a unique representing measure for $M_{d}^{(k)}$ can be explicitly computed using CF7 (see Section 2), and this serves as an approximate representing measure for $M_{d}$. Although a concrete characterization of positivity for $L_{\beta}$ seems unlikely, in view of the ease of detecting flatness (by simply checking the positivity and rank conditions), we are motivated to seek a concrete characterization of membership in $\overline{\mathcal{F}_{d}}$ (cf. Question 1.7 below). To illustrate membership in $\overline{\mathcal{F}_{d}}$, consider the example mentioned just before Theorem 1.2 .

Example 1.5. We have $n=1$ and

$$
M \equiv M_{2}\left(\beta^{(4)}\right)=\left(\begin{array}{ccc}
1 & 1 & 1 \\
1 & 1 & 1 \\
1 & 1 & 2
\end{array}\right)
$$

$M$ is positive semidefinite, but not recursively generated, so $\beta$ has no measure (see Section 2). However, with

$$
M_{2}^{(k)}:=\left(\begin{array}{ccc}
1 & 1 & 1+\frac{1}{k} \\
1 & 1+\frac{1}{k} & 1+\epsilon_{k} \\
1+\frac{1}{k} & 1+\epsilon_{k} & 2
\end{array}\right) \quad\left(\epsilon_{k}=\frac{k^{2}+\sqrt{k^{5}-2 k^{4}-k^{3}}}{k^{3}}\right)
$$


we see that $M_{2}^{(k)}$ is positive and flat, with $\operatorname{rank} M_{2}^{(k)}=\operatorname{rank} M_{1}^{(k)}=2$, and clearly $\lim _{k \rightarrow \infty} M_{2}^{(k)}=M$, so $M_{2}(\beta) \in \overline{\mathcal{F}_{2}}$ and $L_{\beta}$ is positive. (Of course, since $n=1$ in this example, positivity of $L_{\beta}$ also follows from $M \succeq 0$ via sums of squares.) Let $\gamma=\sqrt{k^{3}\left(k^{2}-2 k-1\right)}$ and let $\psi=\sqrt{k^{2}\left(3 k+2 k^{2}+k^{3}-4 \gamma\right)}$. Then (as described in Section 2) the unique representing measure for $M_{2}^{(k)}$, which serves as an approximate representing measure for $M$, is given by $\mu^{(k)}:=\alpha_{1}^{(k)} \delta_{x_{1}^{(k)}}+$ $\alpha_{2}^{(k)} \delta_{x_{2}^{(k)}}$, where $x_{1}^{(k)}=\frac{1}{2 k^{2}}(\gamma-\psi) \longrightarrow 1, x_{2}^{(k)}=\frac{1}{2 k^{2}}(\gamma+\psi) \longrightarrow+\infty, \alpha_{1}^{(k)}=$ $\frac{-2 k^{2}+\gamma+\psi}{2 \psi} \longrightarrow 1, \alpha_{2}^{(k)}=\frac{-2 k^{2}+\gamma-\psi}{2 \psi} \longrightarrow 0$. The fact that $\left\{x_{2}^{(k)}\right\}$ diverges reflects the fact that $\beta$ has no representing measure.

Now suppose $n \geq 1$ and $M_{d}(\beta)=\lim _{k \rightarrow \infty} M_{d}\left(\beta^{[k]}\right)$, where each $\beta^{[k]}$ is a sequence of degree $2 d$ with $M_{d}\left(\beta^{[k]}\right)$ positive and flat. It follows that

$$
M_{d}(\beta) \succeq 0,
$$

and lower semicontinuity of rank ([ $\mathrm{Her}$, Proposition 1.12(i)]) implies that

$$
\operatorname{rank} M_{d}(\beta) \leq \liminf _{k \rightarrow \infty} \operatorname{rank} M_{d}\left(\beta^{[k]}\right)=\liminf _{k \rightarrow \infty} \operatorname{rank} M_{d-1}\left(\beta^{[k]}\right) \leq \rho_{d-1},
$$

whence

$$
\operatorname{rank} M_{d}(\beta) \leq \rho_{d-1} .
$$

For $n=1, d \geq 1$ or $n=d=2$, the main result of [FN2], which follows, characterizes membership in $\overline{\mathcal{F}_{d}}$ in terms of (1.2) and (1.3).

Theorem 1.6 ([FN2]). Let $n=1, d \geq 1$ or $n=d=2 . M_{d} \equiv M_{d}(\beta)$ belongs to $\overline{\mathcal{F}_{d}}$ if and only if $M_{d} \succeq 0$ and rank $M_{d} \leq \rho_{d-1}$. In this case, there exist moment matrices $M_{d}^{(k)} \equiv M_{d}\left(\beta^{[k]}\right)(k \geq 1)$ such that $\lim _{k \rightarrow \infty} M_{d}^{(k)}=M_{d}$ and for each $k$, $\operatorname{rank} M_{d}^{(k)}=\operatorname{rank} M_{d-1}^{(k)}=\operatorname{rank} M_{d}$.

Note also that for $n \geq 1, d=1,(1.2)$ and (1.3) imply that $M_{1}(\beta)$ is flat (with rank 1), so Theorem 1.6 holds in all the cases of Hilbert's theorem; these results motivate the following question.

Question 1.7. Let $n, d \geq 1$. If $M_{d}(\beta) \succeq 0$ and $\operatorname{rank} M_{d} \leq \rho_{d-1}$, does $M_{d}(\beta)$ belong to $\overline{\mathcal{F}_{d}}$ ?

Note that in each case in which Question 1.7 has an affirmative answer, (1.2) and (1.3) provide a simple test for the existence of a representing measure for $\beta^{(2 d-1)}$ (via Theorem 1.2). Theorem 1.1 provides a positive answer to Question 1.7 for $n=2, d=3$; this is perhaps surprising, because several moment theorems which hold within the framework of Hilbert's theorem, e.g., the solution to TMP for $n=d=2$ [CF5], break down in the bivariate degree 6 case (cf. [F]). To further address membership in $\overline{\mathcal{F}_{d}}$, observe the necessay condition arising from Theorem 1.2 that positivity of $L_{\beta}$, including membership of $M_{d}(\beta)$ in $\overline{\mathcal{F}_{d}}$, entails

$$
\beta^{(2 d-1)} \in \mathcal{R}_{n, 2 d-1} \text {. }
$$

Of course, (1.4) is equivalent to TMP for $\beta^{(2 n-1)}$ and is therefore difficult to check in general. Nevertheless, the results of this paper provide some positive evidence concerning the following weaker version of Question 1.7 
Question 1.8. Let $n, d \geq 1$. If $M_{d} \succeq 0$, $\operatorname{rank} M_{d} \leq \rho_{d-1}$, and $\beta^{(2 d-1)}$ has a representing measure, is $\bar{M}_{d}$ a limit of positive flat moment matrices?

Let $M_{d} \equiv M_{d}(\beta)$ denote a bivariate moment matrix of degree $2 d$, and consider the block matrix decomposition

$$
M_{d} \equiv\left(\begin{array}{cc}
M_{d-1} & B_{d} \\
B_{d}^{T} & C_{d}
\end{array}\right)
$$

thus $M_{d-1}$ and $B_{d}$ together contain the data in $\beta^{(2 d-1)}$, and $C_{d}$ is a Hankel matrix comprised of the data of degree $2 d$. As we discuss in Section $2, M_{d}$ is positive semidefinite if and only if i) $M_{d-1} \succeq 0$, ii) there exists a matrix $W$ such that $B_{d}=M_{d-1} W$, and iii) $C_{d} \succeq C^{b} \equiv C_{d}^{b}:=W^{T} M_{d-1} W$, i.e., $\Delta^{b} \equiv C_{d}-C_{d}^{b} \succeq 0$. Let $\rho_{d}=\operatorname{dim} \mathbb{R}[x, y]_{d}\left(=\frac{(d+1)(d+2)}{2}\right)$, which coincides with the size of $M_{d}$; thus, $r \equiv \operatorname{rank} M_{d-1} \leq \rho_{d-1}$. Now suppose $\beta^{(2 d-1)}$ has a $\kappa$-atomic representing measure $\mu$; then $M_{d}[\mu]$ is of the form

$$
M_{d}[\mu]=\left(\begin{array}{cc}
M_{d-1} & B_{d} \\
B_{d}^{T} & C_{d}[\mu]
\end{array}\right) .
$$

Let $\Delta \equiv \Delta[\mu]:=C_{d}-C_{d}[\mu], s=\operatorname{rank} \Delta[\mu], \rho[\mu]:=s+\kappa$, and

$$
M_{\Delta}:=\left(\begin{array}{cc}
0 & 0 \\
0 & \Delta
\end{array}\right) .
$$

We now state our main result.

Theorem 1.9. Let $n=2, d \geq 1$. Suppose that $M_{d}(\beta) \succeq 0$ and that $\beta^{(2 d-1)}$ has a $\kappa$-atomic representing measure $\mu$. If $\Delta \equiv \Delta[\mu] \succeq 0$ and $\rho[\mu] \leq \rho_{d-1}$, then $M_{d} \in \overline{\mathcal{F}_{d}}$. Moreover, if $\rho[\mu] \leq \tau \leq \rho_{d-1}$, then there exists a sequence of positive flat moment matrices, $\left\{M_{d}^{(k)}\right\}_{k=1}^{\infty}$, such that $M_{d}(\beta)=\lim _{k \rightarrow \infty} M_{d}^{(k)}$ and, for each $k$, $\operatorname{rank} M_{d}^{(k)}=\operatorname{rank} M_{d-1}^{(k)}=\tau$.

We note two basic cases where Theorem 1.9 applies. For $M_{d} \succeq 0$, let

$$
M^{\mathrm{b}}:=\left(\begin{array}{cc}
M_{d-1} & B_{d} \\
B_{d}^{T} & C^{\mathrm{b}}
\end{array}\right)
$$

and

so that

$$
M_{\Delta^{b}}:=\left(\begin{array}{cc}
0 & 0 \\
0 & \Delta^{b}
\end{array}\right)
$$

$$
M_{d}=M^{\mathrm{b}}+M_{\Delta^{\mathrm{b}}} .
$$

Let $r=\operatorname{rank} M_{d-1}$ and $s^{b}=\operatorname{rank} \Delta^{b}$. Then $\rho \equiv \operatorname{rank} M_{d}=r+s^{b}$ and $\operatorname{rank} M^{b}=$ $r$. For the cases covered by Theorem 1.6, we showed in FN2 that $C^{b}$ is always Hankel; thus, Theorem 1.6 can be derived from the following result.

Corollary 1.10. The conclusions of Theorem 1.9 hold if $M_{d} \succeq 0, \rho \equiv \operatorname{rank} M_{d} \leq$ $\rho_{d-1}$, and $C^{\text {b }}$ is Hankel.

Proof. Suppose $M_{d} \succeq 0$ and rank $M_{d} \leq \rho_{d-1}$. If $C^{b}$ is a Hankel matrix, then $M^{b}$ is a flat moment matrix extension of $M_{d-1}$ using the data in $B_{d}$. Theorem 1.4 thus shows that $M^{b}$ has a unique representing measure $\mu$, which is $r$-atomic $\left(r \equiv \operatorname{rank} M_{d-1}\right)$. Thus, the hypotheses of Theorem 1.9 hold with $\kappa=r, \Delta=\Delta^{b}$, $s=s^{b}, \rho[\mu]=r+s=\rho \leq \rho_{d-1}$; note from iii) above that $\Delta \succeq 0$ since $M_{d} \succeq 0$. 
In Example 2.1, (1.2) and (1.3) hold, but $C^{b}$ is not Hankel; however, we show in Section 4 that this example falls within the scope of the next result.

Corollary 1.11. The conclusions of Theorem 1.9 hold if $M_{d}$ has a $\kappa$-atomic representing measure $\mu$ with $\kappa \leq \rho_{d-1}$; in particular, this applies if $M_{d} \succeq 0$, rank $M_{d} \leq \rho_{d-1}$, and $M_{d}$ admits a flat extension $M_{d+1}$.

Proof. For $\mu$ satisfying the hypothesis, $\Delta[\mu]=0, s=0, \rho[\mu]=\kappa \leq \rho_{d-1}$, so Theorem 1.9 applies. In the flat extension case, Theorem 1.4 implies the existence of $\mu$ with $\kappa \equiv$ card supp $\mu=\operatorname{rank} M_{d} \leq \rho_{d-1}$.

If we ignore the calculations of ranks in the proof of Theorem 1.9, we arrive at the following criterion for positivity of $L_{\beta}$.

Proposition 1.12. If $M_{d}(\beta) \succeq 0$ and $\beta^{(2 d-1)}$ has a finitely atomic representing measure $\mu$ such that $\Delta[\mu] \succeq 0$, then $L_{\beta}$ is positive.

Section 2 concerns positive moment matrices, including a geometric characteriztion of flatness (Theorem 2.7) that we require in the sequel. Section 3 concerns determining sequences, which provide a tool for relating the rank of a moment matrix to the geometry of the support of a representing measure. Using Proposition 3.1 and particular determining sequences that we describe in Section 3, we prove Theorem 1.9 and Proposition 1.12 in Section 4. The proof of Theorem 1.1 is a synthesis of these and other results and appears in Section 5. Theorem 1.1 also yields a new solution to the singular quartic moment problem (Proposition 5.13). The proofs of Theorem 1.1 and of several examples that we present below depend in part on calculations using the computer algebra system in Mathematica; in the sequel we refer to these as symbolic calculations.

We conclude this section by noting an application of Theorem 1.9 to polynomial optimization on $\mathbb{R}^{2}$. For $p \in \mathbb{R}[x, y]$, the Optimization Problem entails estimating

$$
p_{*}:=\inf _{(x, y) \in \mathbb{R}^{2}} p(x, y) .
$$

We recall the moment relaxations for (1.6) introduced by J.-B. Lasserre [Las]. For $t \geq 1$, we define the $t$-th Lasserre relaxation by

$$
p_{t}:=\inf \left\{L_{y}(p): y \equiv y^{(2 t)}, y_{00}=1, M_{t}(y) \succeq 0\right\} .
$$

It is not difficult to check that $p_{t} \leq p_{*}$ and that for $t^{\prime} \geq t, p_{t^{\prime}} \geq p_{t}$; thus, $\left\{p_{t}\right\}$ is convergent, and $p^{m o m} \equiv \lim _{t \rightarrow \infty} p_{t} \leq p_{*}$. For fixed $t$, the infimum in (1.6) is not necessarily attained. Assuming that the infimum is attained, at some optimal sequence $y_{*} \equiv y_{*}^{(2 t)}$, we seek criteria which imply that $L_{y_{*}}(p)=p_{*}$, so that we have finite convergence of $\left\{p_{s}\right\}$ to $p_{*}$ at stage $s=t$. A basic result of [Las] shows that this is the case if $M_{t}\left(y_{*}\right)$ is flat, and this concrete condition is used as the stopping criterion in the optimization program in HeLa]. In [FN2, Theorem 1.5] we showed that convergence at stage $t$ occurs, more generally, if $L_{y_{*}}$ is positive. Thus, Corollary 1.10 provides a broader concrete condition for finite convergence than flatness, namely, $\operatorname{rank} M_{t}\left(y_{*}\right) \leq \rho_{t-1}$ and $C_{t}^{b}\left(y_{*}\right)$ Hankel.

\section{Positive moment matrices}

In this section we present some results concerning positive moment matrices that will be used in the sequel. We begin, more generally, with a real symmetric block 
matrix of the form

$$
M=\left(\begin{array}{cc}
A & B \\
B^{T} & C
\end{array}\right)
$$

It is well known that $M$ is positive semidefinite if and only if $A \succeq 0, B=A W$ for some matrix $W$, and $C \succeq W^{T} A W\left(=B^{T} W\right)$ (cf. [CF1]). In this case, let $\Delta \equiv$ $C-W^{T} A W$ denote the Schur complement, so that $\operatorname{rank} M=\operatorname{rank} A+\operatorname{rank} \Delta$. We have $\operatorname{rank} M=\operatorname{rank} A$, and we say that $M$ is a flat extension of $A$ if and only if $C=W^{T} A W$. Flat extensions are uniquely determined by $A$ and $B$, for if there are matrices $W$ and $V$ such that $A W=B=A V$, then $W^{T} A W-V^{T} A V=$ $W^{T} A V-V^{T} A V=(A W-A V)^{T} V=0$. Given $A \succeq 0$ and $B=A W$ as above, if we set $C^{b}=W^{T} A W$ and

$$
M^{b}=\left(\begin{array}{cc}
A & B \\
B^{T} & C^{b}
\end{array}\right)
$$

then clearly $M^{b}$ is a positive, flat extension of $A$.

Let us denote the positive $n$-variable moment matrix $M_{d} \equiv M_{d}(\beta)$ by

$$
M_{d}(\beta)=\left(\begin{array}{cc}
M_{d-1}(\beta) & B_{d} \\
B_{d}^{T} & C_{d}
\end{array}\right)
$$

with $B_{d}=M_{d-1} W$. In this setting, we sometimes denote $C^{\text {b }}$ by $C_{d}^{b}$. Following CF2, CF7, we say that $M_{d}$ is flat if rank $M_{d}=\operatorname{rank} M_{d-1}$, i.e., $C_{d}=C_{d}^{b}$. Theorem 1.4 implies that in this case $M_{d}$ has a unique representing measure which is $\left(\operatorname{rank} M_{d}\right)$-atomic. For $M_{d} \succeq 0$, a rank-preserving extension $M_{d+1}$ is said to be a flat extension; in this case, $M_{d+1} \succeq 0$ and $M_{d+1}$ is itself flat. In the sequel, if $\mu$ is a positive Borel measure with convergent moments $\beta \equiv \beta^{(2 d)}$, we sometimes denote $M_{d}(\beta)$ by $M_{d}[\mu]$; moreover, for a moment matrix $M_{d}(\beta)$, we sometimes refer to a representing measure for $\beta$ as a representing measure for $M_{d}(\beta)$.

Let $n=2$ and suppose $M_{d}(\beta) \succeq 0$, so that $B_{d}=M_{d-1}(\beta) W$ for some matrix $W$ (as above). Since $n=2, C_{d}$ is a Hankel matrix; i.e., $C_{d}$ is constant on crossdiagonals. We note that in general $C^{b} \equiv W^{T} M_{d-1} W$ need not be Hankel. We illustrate this in the following example of [FN2], which we will analyze further in Section 4.

Example 2.1. Consider the positive moment matrix $M_{3}(\beta)$ defined by

$$
M_{3}(\beta)=\left(\begin{array}{cccccccccc}
1 & 0 & 0 & 1 & 0 & 1 & 0 & 0 & 0 & 0 \\
0 & 1 & 0 & 0 & 0 & 0 & 1 & 0 & 1 & 0 \\
0 & 0 & 1 & 0 & 0 & 0 & 0 & 1 & 0 & 2 \\
1 & 0 & 0 & 1 & 0 & 1 & 0 & 0 & 0 & 0 \\
0 & 0 & 0 & 0 & 1 & 0 & 0 & 0 & 0 & 0 \\
1 & 0 & 0 & 1 & 0 & 2 & 0 & 0 & 0 & 0 \\
0 & 1 & 0 & 0 & 0 & 0 & 1 & 0 & 1 & 0 \\
0 & 0 & 1 & 0 & 0 & 0 & 0 & 1 & 0 & 2 \\
0 & 1 & 0 & 0 & 0 & 0 & 1 & 0 & 2 & 0 \\
0 & 0 & 2 & 0 & 0 & 0 & 0 & 2 & 0 & 4
\end{array}\right) .
$$


Then $B_{3}=M_{2} W$, where

$$
W:=\left(\begin{array}{cccc}
0 & 0 & 1 & 0 \\
1 & 0 & 1 & 0 \\
0 & 1 & 0 & 2 \\
0 & 0 & 0 & 0 \\
0 & 0 & 0 & 0 \\
0 & 0 & 0 & 0
\end{array}\right),
$$

so $C^{b} \equiv W^{T} M_{2} W=\left(\begin{array}{cccc}1 & 0 & 1 & 0 \\ 0 & 1 & 0 & 2 \\ 1 & 0 & 1 & 0 \\ 0 & 2 & 0 & 4\end{array}\right)$ and $\Delta \equiv C_{3}-C^{b}=\left(\begin{array}{cccc}0 & 0 & 0 & 0 \\ 0 & 0 & 0 & 0 \\ 0 & 0 & 1 & 0 \\ 0 & 0 & 0 & 0\end{array}\right)$.

In [FN2 we used ad hoc methods to show that $M_{3} \in \overline{\mathcal{F}}_{3}$. In Section 4 we will establish membership in $\overline{\mathcal{F}}_{3}$ based on Corollary 1.11 .

In the sequel we denote the successive columns of $M_{d}(\beta)$ by $X^{i}(|i| \leq d)$ in degreelexicographic order. Let $p \in \mathbb{R}[x]_{d}, p=\sum a_{i} x^{i}$. We define a corresponding element of $\operatorname{Col} M_{d}(\beta)$, the column space of $M_{d}(\beta)$, by $p(X):=\sum a_{i} X^{i}\left(=M_{d} \widehat{p}\right)$. Thus, each column dependence relation in $M_{d}(\beta)$ can be expressed as $p(X)=0$ for some $p \in \mathbb{R}[x]_{d}, p \not \equiv 0$. Following [CF2], CF7, we say that $M_{d}(\beta)$ is recursively generated if $p, q, p q \in \mathbb{R}[x]_{d}, p(X)=0 \Longrightarrow(p q)(X)=0$. Positivity and recursiveness are necessary conditions for $\beta$ to have a representing measure [CF7, and for $n=1$ these conditions are also sufficient [CF1] (cf. Theorem 2.3). In the sequel, to construct examples, we will employ the following result without further reference.

Theorem 2.2 (Structure Theorem [CF2, CF7]). If $M_{d}(\beta)$ is positive semidefinite, then the following properties hold:

i) $M_{d-1}(\beta)$ is recursively generated.

ii) If $p \in \mathcal{P}_{d-1}$ and $p(X)=0$ in $\operatorname{Col} M_{d-1}$, then $p(X)=0$ in Col $M_{d}$.

iii) If $p \in \mathcal{P}_{d}$ satisfies $p(X)=0$ in $C o l M_{d}$ and $q \in \mathcal{P}_{d}$ satisfies deg $p q \leq d$, then $(p q)(X)=0$ in Col $\left(M_{d-1}(y) \quad B_{d}\right)$. Further, if $M_{d}$ is flat, then $(p q)(X)=0$ in $\mathrm{Col} M_{d}$.

For $n=1$, we will denote a truncated moment sequence of degree $2 d$ by $y^{(2 d)}=$ $\left\{y_{0}, \ldots, y_{2 d}\right\}$. Then $M_{d}(y)$ is a Hankel matrix, which we henceforth denote by $H_{d} \equiv H_{d}(y):=\left(y_{a+b}\right)_{0 \leq a, b \leq d}$. In this case, Theorem 2.2 may be expressed as follows.

Theorem 2.3 ([CF1]). Suppose $H_{d} \equiv H_{d}(y)$ is a positive Hankel matrix, $y_{0}>0$. If $H_{d} \succ 0$ (positive definite), then $H_{d}$ admits infinitely many distinct flat extensions and corresponding $(d+1)$-atomic representing measures. If $H_{d}$ is singular, let $r:=\min \left\{s: 1 \leq s \leq d: H_{s}\right.$ is singular $\}$. Let $v=\left(y_{r}, \ldots, y_{2 r-1}\right)^{T}$ and let $c \equiv\left(c_{0}, \ldots, c_{r-1}\right)^{T}=M_{r-1}^{-1} v$. Then $(a) y_{j}=c_{0} y_{j-r}+\cdots+c_{r-1} y_{j-1}(r \leq j \leq 2 d-1)$ and (b) $y_{2 d} \geq c_{0} y_{2 d-r}+\cdots+c_{r-1} y_{2 d-1}$.

Further, the following are equivalent: i) y has a representing measure; ii) equality holds in (b) (above); iii) $H_{d}$ is recursively generated; iv) rank $H_{d}=r$; v) $H_{d}$ is a flat extension of $H_{r-1}$. There is strict inequality in (b) (above) $\Longleftrightarrow$ rank $H_{d}=r+1$.

Recall the variety associated with $\beta$ and $M_{d}(\beta)$, defined by

$$
\mathcal{V} \equiv \mathcal{V}(\beta):=\bigcap_{p \in \mathbb{R}[x]_{d}, p(X)=0} \mathcal{Z}(p)
$$


where $\mathcal{Z}(p):=\left\{x \in \mathbb{R}^{n}: p(x)=0\right\}$. We will repeatedly use the following result.

Proposition 2.4 ([CF2, CF7, Prop. 2.10, Cor. 2.12]). If $\beta$ has a representing measure $\mu$, then supp $\mu \subseteq \mathcal{V}(\beta)$. Furthermore,

i) $\operatorname{rank} M_{d} \leq$ card supp $\mu \leq \operatorname{card} \mathcal{V}(\beta)$;

ii) for $p \in \mathbb{R}[x]_{d}, p(X)=0$ in Col $M_{d}$ (equivalently, $\left.M_{d} \widehat{p}=0\right) \Longleftrightarrow p \mid \operatorname{supp} \mu \equiv 0$.

The next result shows how the variety can be used to construct the unique representing measure corresponding to a flat extension.

Theorem 2.5 ([CF7, Theorem 1.2]). Suppose $M_{d} \succeq 0$ admits a flat extension $M_{d+1}(\widetilde{\beta})$, i.e., $\operatorname{rank} M_{d+1}(\widetilde{\beta})=\operatorname{rank} M_{d}$. Then $\operatorname{card} \mathcal{V}(\widetilde{\beta})=\rho \equiv \operatorname{rank} M_{d}$, say $\mathcal{V}(\widetilde{\beta})=\left\{w_{1}, \ldots, w_{\rho}\right\}$. Let $X^{i_{1}}, \ldots, X^{i_{\rho}}$ denote a basis for the column space of $M_{d}$, define $p_{j}(x):=x^{i_{j}} \in \mathbb{R}[x]_{d}$, and let $\omega=\left(\beta_{i_{1}}, \ldots, \beta_{i_{\rho}}\right)$. Then the Vandermonde matrix $V \equiv\left(p_{j}\left(w_{k}\right)\right)_{1 \leq j, k \leq \rho}$ is invertible, and we define $\alpha \equiv\left(\alpha_{1}, \ldots, \alpha_{\rho}\right)$ by $\alpha^{T}:=$ $V^{-1} \omega^{T}$. The unique representing measure for $\widetilde{\beta}$ is given by

$$
\mu:=\sum_{i=1}^{\rho} \alpha_{i} \delta_{w_{i}}
$$

We now consider the moment matrix determined by a finitely atomic measure. For $w \equiv\left(x_{1}, \ldots, x_{n}\right) \in \mathbb{R}^{n}$, let $\zeta \equiv \zeta[w]=\left(1, x_{1}, x_{2}, \ldots, x_{1}^{d}, x_{1}^{d-1} x_{2}, \ldots\right.$, $\left.x_{n-1} x_{n}^{d-1}, x_{n}^{d}\right)$. In the sequel, $\delta_{\{w\}}$ denotes the atomic measure with support $\{w\}$, whose moment sequence $\beta \equiv \beta^{(2 d)}$ satisfies $\beta_{i}=x_{1}^{i_{1}} \cdots x_{n}^{i_{n}}\left(i \equiv\left(i_{1}, \ldots, i_{n}\right)\right.$, $|i| \leq 2 d)$. As noted in $\mathrm{Lau}, M_{d}\left[\delta_{\{w\}}\right] \equiv M_{d}(\beta)=\zeta^{T} \zeta$, so $\operatorname{rank} M_{d}\left[\delta_{\{w\}}\right]=1$. It follows from subadditivity of rank that if $\mu$ is a $t$-atomic measure, $\mu \equiv \sum_{i=1}^{t} \alpha_{i} \delta_{\left\{w_{i}\right\}}$ with each $\alpha_{i}>0$, then

$$
\operatorname{rank} M_{d}[\mu]=\operatorname{rank} \sum_{i=1}^{t} \alpha_{i} M_{d}\left[\delta_{\left\{w_{i}\right\}}\right] \leq \sum_{i=1}^{t} \operatorname{rank} \alpha_{i} M_{d}\left[\delta_{\left\{w_{i}\right\}}\right]=t .
$$

In Theorem 2.7, we present a geometric criterion for flatness that we will use in the proof of Theorem [1.9. We begin with a lower estimate for rank $M_{d}$ which complements the upper estimate in Proposition 2.4 i). Given $\mathcal{P} \equiv\left\{p_{1}, \ldots, p_{t}\right\} \subset$ $\mathbb{R}[x]_{d}$ and points $w \equiv\left\{w_{i}\right\}_{i=1}^{t} \subset \mathbb{R}^{n}$, let $V \equiv V[\mathcal{P} ; w]$ denote the Vandermonde-type matrix

$$
\left(\begin{array}{ccc}
p_{1}\left(w_{1}\right) & \cdots & p_{t}\left(w_{1}\right) \\
\vdots & \vdots & \vdots \\
p_{1}\left(w_{t}\right) & \cdots & p_{t}\left(w_{t}\right)
\end{array}\right)
$$

Proposition 2.6. If $w \equiv\left\{w_{1}, \ldots, w_{t}\right\} \subseteq \mathcal{V}(\beta), \mathcal{P} \equiv\left\{p_{1}, \ldots, p_{t}\right\} \subset \mathbb{R}[x]_{d}$, and $V \equiv V[\mathcal{P} ; w]$ is invertible, then rank $M_{d}(\beta) \geq t$. If $M_{d}$ has a t-atomic representing measure $\mu$, supp $\mu=w \equiv\left\{w_{1}, \ldots, w_{t}\right\}$, then rank $M_{d}=t \Longleftrightarrow$ there exist polynomials $\mathcal{P} \equiv\left\{p_{1}, \ldots, p_{t}\right\} \subset \mathbb{R}[x]_{d}$ such that $V[\mathcal{P} ; w]$ is invertible.

Proof. Let $v:=\rho_{d}$; since $V$ is invertible, $\mathcal{P}$ is independent, so we may extend $\mathcal{P}$ to a basis $\left\{p_{j}\right\}_{j=1}^{v}$ for $\mathbb{R}[x]_{d}$. Suppose $p \equiv \sum_{i=1}^{v} c_{i} p_{i} \in \mathbb{R}[x]_{d}$ and $\widehat{p} \in \operatorname{ker} M_{d}$; then $p \mid \mathcal{V}(\beta) \equiv 0$, so $p\left(w_{j}\right)=0(1 \leq j \leq t)$. 
We first consider the case when $t<v$. Setting

$$
\widehat{V}=\left(\begin{array}{cccccc}
p_{1}\left(w_{1}\right) & \cdots & p_{t}\left(w_{1}\right) & p_{t+1}\left(w_{1}\right) & \cdots & p_{v}\left(w_{1}\right) \\
\vdots & \vdots & \vdots & \vdots & \vdots & \vdots \\
p_{1}\left(w_{t}\right) & \cdots & p_{t}\left(w_{t}\right) & p_{t+1}\left(w_{t}\right) & \cdots & p_{v}\left(w_{t}\right)
\end{array}\right) \equiv\left(\begin{array}{ll}
V & \widetilde{V}
\end{array}\right)
$$

and $c \equiv\left(c_{1}, \ldots, c_{v}\right)^{T}$, we have

$$
\widehat{V} c=0 .
$$

Now $V$ is invertible, so multiplying $(2.7)$ by $V^{-1}$ on the left, we obtain $K c=0$, with $K$ of the form $\left(\begin{array}{ll}I_{t} & D\end{array}\right)$, where $I_{t}$ denotes the $t \times t$ identity matrix and $D:=V^{-1} \widetilde{V}$ is of the form $D_{t \times(v-t)} \equiv\left(d_{i j}\right)_{1 \leq i \leq t, 1 \leq j \leq v-t}$. We thus see that

$$
c_{i}+d_{i 1} c_{t+1}+\cdots+d_{i, v-t} c_{v}=0(1 \leq i \leq t) .
$$

Now,

$$
\begin{aligned}
p= & \left(-d_{1,1} c_{t+1}-\cdots-d_{1, v-t} c_{v}\right) p_{1}+\cdots+\left(-d_{t, 1} c_{t+1}-\cdots-d_{t, v-t} c_{v}\right) p_{t} \\
& +c_{t+1} p_{t+1}+\cdots+c_{v} p_{v} \\
= & c_{t+1}\left(-d_{1,1} p_{1}-\cdots-d_{t, 1} p_{t}+p_{t+1}\right)+c_{t+2}\left(-d_{1,2} p_{1}-\cdots-d_{t, 2} p_{t}+p_{t+2}\right) \\
& +\cdots+c_{v}\left(-d_{1, v-t} p_{1}-\cdots-d_{t, v-t} p_{t}+p_{v}\right) .
\end{aligned}
$$

The preceding calculation shows that $k e r M_{d}$ is spanned by the $v-t$ vectors $\widehat{\eta}_{j}$ $(1 \leq j \leq v-t)$, where

$$
\eta_{j}:=-d_{1, j} p_{1}-\cdots-d_{t, j} p_{t}+p_{t+j} .
$$

Thus, $\operatorname{dim} \operatorname{ker} M_{d} \leq v-t$, and it follows that rank $M_{d}=\rho_{d}-\operatorname{dim} \operatorname{ker} M_{d} \geq$ $\rho_{d}-(v-t)=t$.

In the case when $t=v$, we set $\widehat{V}=V$ and conclude from (2.7) that $\widehat{c}=0$, whence $\operatorname{ker} M_{d}=\{0\}$. Thus, $\operatorname{rank} M_{d}=\rho_{d}=v=t$.

Next, suppose $\mu$ is a representing measure, supp $\mu=w \equiv\left\{w_{i}\right\}_{i=1}^{t}$. From Proposition 2.4,w $\subseteq \mathcal{V}(\beta)$ and rank $M_{d} \leq t$. If there exists $\mathcal{P} \equiv\left\{p_{i}\right\}_{i=1}^{t} \subset \mathbb{R}[x]_{d}$ such that $V[\mathcal{P} ; w]$ is invertible, then the above argument implies that rank $M_{d}=t$. Conversely, suppose rank $M_{d}=t$, and let $\mathcal{B} \equiv\left\{X^{i_{1}}, \ldots, X^{i_{t}}\right\}$ denote a basis for $\operatorname{Col} M_{d}$. Let $\mathcal{P}=\left\{x^{i_{j}}\right\}_{j=1}^{t} \subset \mathbb{R}[x]_{d}$. Suppose $p:=\sum_{j=1}^{t} c_{j} x^{i_{j}}, p \not \equiv 0$, and $V[\mathcal{P} ; w] \widehat{p}=0$, i.e., $p \mid \operatorname{supp} \mu \equiv 0$. Since $\mu$ is a representing measure for $M_{d}$, Proposition 2.4 ii) implies $p(X)=0$ in $C o l M_{d}$, contradicting the independence of $\mathcal{B}$; thus $V[\mathcal{P} ; w]$ is invertible.

Theorem 2.7. $M_{d} \succeq 0$ is flat if and only if there exist a t-atomic representing measure $\mu$ for $M_{d}$, supp $\mu=w \equiv\left\{w_{1}, \ldots, w_{t}\right\} \subset \mathbb{R}^{n}$, and polynomials $\mathcal{P} \equiv$ $\left\{p_{1}, \ldots, p_{t}\right\} \subset \mathbb{R}[x]_{d-1}$, such that $V[\mathcal{P} ; w]$ is invertible.

Proof. The "only if" direction follows immediately from Theorem 2.5] applied to the flat extension $M_{d}$ of $M_{d-1}$. For the converse, it follows from Proposition 2.6 (applied to $M_{d-1}$ ), and from (2.5), that $t=\operatorname{rank} M_{d-1} \leq \operatorname{rank} M_{d} \leq t$, so $M_{d}$ is flat.

We conclude this section with a change of variables result for $n=2$ that we will use in Section 5 to simplify certain moment matrices. An analogous change of variables for the truncated complex moment problem appears in CF5, Proposition 
1.7], but here we require a stronger version. Consider the degree-one map $\mathcal{T}: \mathbb{R}^{2} \mapsto$ $\mathbb{R}^{2}$ defined by $\mathcal{T}(x, y)=(\widetilde{x}, \widetilde{y}) \equiv(a+\alpha x+\gamma y, b+\delta x+\lambda y)$, where $\alpha \lambda-\gamma \delta \neq 0$ (which insures that $\mathcal{T}$ is a bijection). Given $\beta \equiv \beta^{(2 d)}$, let $L_{\beta}$ denote its Riesz functional. We define a multisequence $\widetilde{\beta} \equiv \widetilde{\beta}^{(2 d)}$ by $\widetilde{\beta}_{i j}=L_{\beta}\left(\widetilde{x}^{i} \widetilde{y}^{j}\right)(i, j \geq 0$, $i+j \leq 2 d)$. Let $\widetilde{M}_{d} \equiv M_{d}(\widetilde{\beta})$ denote the moment matrix for $\widetilde{\beta}$ and let $L_{\widetilde{\beta}}$ denote its Riesz functional. For $p \in \mathbb{R}[x, y]_{2 d}, p(x, y) \equiv \sum a_{i j} x^{i} y^{j}$, we have $L_{\widetilde{\beta}}(p)=$ $L_{\beta}(p(\widetilde{x}, \widetilde{y}))=L_{\beta}\left(\sum a_{i j}(a+\alpha x+\gamma y)^{i}(b+\delta x+\lambda y)^{j}\right)$. Let $\mathcal{P}_{d}$ denote the space of polynomial vectors of degree at most $d$, i.e., $\mathcal{P}_{d}=\left\{\widehat{p}: p \in \mathbb{R}[x, y]_{d}\right\}$, and define a matrix $J \equiv J_{d}: \mathcal{P}_{d} \mapsto \mathcal{P}_{d}$ by $J \widehat{p}:=\widehat{p(\widetilde{x}, \widetilde{y})}\left(p \in \mathbb{R}[x, y]_{d}\right)$; for example, $J \widehat{x} \equiv J(0,1,0,0, \ldots, 0)^{T}=(a, \alpha, \gamma, 0, \ldots, 0)^{T}$.

Proposition 2.8. i) $\widetilde{M}_{d}=J^{T} M_{d} J$;

ii) $J$ is invertible;

iii) $\widetilde{M}_{d} \succeq 0 \Longleftrightarrow M_{d} \succeq 0$;

iv) $\operatorname{rank} \widetilde{M}_{d}=\operatorname{rank} M_{d}$.

v) The formula $\mu=\widetilde{\mu} \circ \mathcal{T}$ is a one-to-one correspondence between representing measures for $\beta$ and $\widetilde{\beta}$, which preserves measure class and cardinality of support; moreover, $\mathcal{T}(\operatorname{supp} \mu)=\operatorname{supp} \widetilde{\mu}$.

vi) $\widetilde{M}_{d}(\succeq 0)$ admits a flat extension if and only if $M_{d}(\succeq 0)$ admits a flat extension.

vii) For $p \in \mathbb{R}[x, y]_{d}$, define $q(\widetilde{x}, \widetilde{y}):=p \circ \mathcal{T}^{-1}(\widetilde{x}, \widetilde{y}) \in \mathbb{R}[\widetilde{x}, \widetilde{y}]_{d}$. Then in Col $\widetilde{M}_{d}$, $q(\tilde{X}, \widetilde{Y})=J^{T} p(X, Y)$. In particular, $p(X, Y)=0$ if and only if $q(\tilde{X}, \tilde{Y})=0$, and $\mathcal{V}(\widetilde{\beta})=\mathcal{T}(\mathcal{V}(\beta))$; further, $\widetilde{M}_{d}$ is recursively generated if and only if $M_{d}$ is recursively generated.

viii) $\widetilde{M}_{d} \in \mathcal{F}_{d}$ if and only if $M_{d} \in \mathcal{F}_{d}$.

ix) Let $\tau$ satisfy $1 \leq \tau \leq \rho_{d-1}$ and let $\mathcal{F}_{d, \tau}=\left\{M_{d} \in \mathcal{F}_{d}:\right.$ rank $\left.M_{d}=\tau\right\}$. Then $\widetilde{M}_{d} \in \overline{\mathcal{F}_{d, \tau}}$ if and only if $M_{d} \in \overline{\mathcal{F}_{d, \tau}}$.

x) For $M_{d} \succeq 0, \widetilde{M_{d}^{b}} \equiv J^{T} M_{d}^{b} J=\left(\widetilde{M_{d}}\right)^{b}$; in particular, $C_{d}^{b}$ is Hankel in $M_{d}^{b}$ if and only if the $C_{d}^{b}$ block of $\left(\widetilde{M}_{d}\right)^{b}$ is Hankel.

Proof. The proofs of i)-vi) are direct analogues of those of the corresponding parts of [CF5, Proposition 1.7], so we omit the details. For vii), we have $q(\widetilde{X}, \widetilde{Y})=\widetilde{M}_{d} \widehat{q}=$ $\widetilde{M}_{d} p \widehat{\circ \mathcal{T}^{-1}}=J^{T} M_{d}\left(\left(J p \widehat{\circ \mathcal{T}^{-1}}\right)\right)=J^{T} M_{d}\left(p \circ \widehat{\mathcal{T}^{-1}} \circ \mathcal{T}\right)=J^{T} M_{d} \widehat{p}=J^{T} p(X, Y)$. Since $J$ is invertible, it follows that $q(\widetilde{X}, \widetilde{Y})=0 \Longleftrightarrow p(X, Y)=0$; the other conclusions follow similarly. For viii), first note that, relative to $\widetilde{\beta}$, for $1 \leq k \leq d$, we also have the subsequence $\widetilde{\beta}^{(2 k)}$ and its moment matrix $\widetilde{M}_{k}$ (a submatrix of $\left.\widetilde{M}_{d}\right)$. For $p \in \mathbb{R}[x, y]_{d}$, $\operatorname{deg} p(x, y)=\operatorname{deg} p(\widetilde{x}, \widetilde{y})$, so $J$ maps $\mathcal{P}_{k}$ onto itself and the map $J_{k}$ associated to $\widetilde{\beta}^{(2 k)}$ satisfies $J_{k}=J \mid \mathcal{P}_{k}$. Each $J_{k}$ is invertible, and $\widetilde{M}_{k}=J_{k}^{T} M_{k} J_{k}$. In particular, $\operatorname{rank} \widetilde{M}_{d-1}=\operatorname{rank} M_{d-1}$. It now follows from iii) and iv) that $\widetilde{M}_{d}$ is positive and flat if $M_{d}$ is positive and flat. The converse follows by symmetry (using the transform $\mathcal{T}^{-1}$ ). For ix), suppose $M_{d}=\lim _{k \rightarrow \infty} M_{d}^{[k]}$, where each $M_{d}^{[k]}$, corresponding to $\beta^{[k]}$ (a sequence of degree $2 d$ ), is positive and flat, with $\operatorname{rank} M_{d}^{[k]}=\tau$. Then $\widetilde{M}_{d} \equiv J^{T} M_{d} J=\lim _{k \rightarrow \infty} J^{T} M_{d}^{[k]} J$, and iii)-iv) and viii) imply that each $J^{T} M_{d}^{[k]} J$ is positive and flat, with $\operatorname{rank} J^{T} M_{d}^{[k]} J=\tau$. Thus 
$M_{d} \in \overline{\mathcal{F}_{d, \tau}} \Longrightarrow \widetilde{M}_{d} \in \overline{\mathcal{F}_{d, \tau}}$, and the converse follows by symmetry (again using the transform $\mathcal{T}^{-1}$ ).

For x), since $M_{d} \succeq 0$, there is a matrix $W$ such that

$$
M_{d}=\left(\begin{array}{cc}
M_{d-1} & M_{d-1} W \\
W^{T} M_{d-1} & C_{d}
\end{array}\right), C_{d} \succeq C_{d}^{b} \equiv W^{T} M_{d-1} W,
$$

and

Denoting

$$
M_{d}^{b}=\left(\begin{array}{cc}
M_{d-1} & M_{d-1} W \\
W^{T} M_{d-1} & W^{T} M_{d-1} W
\end{array}\right)
$$

we have

$$
J \equiv J_{d}=\left(\begin{array}{cc}
J_{d-1} & K \\
0 & L
\end{array}\right)
$$

$$
\widetilde{M}_{d} \equiv J^{T} M_{d} J=\left(\begin{array}{cc}
\widetilde{M}_{d-1} & \widetilde{B}_{d} \\
\widetilde{B}_{d}^{T} & \widetilde{C}_{d}
\end{array}\right),
$$

where $\widetilde{M}_{d-1}=J_{d-1}^{T} M_{d-1} J_{d-1}$ and $\widetilde{B}_{d}=\widetilde{M}_{d-1} \widetilde{W}$, with $\widetilde{W}=J_{d-1}^{-1}(K+W L)$. Thus

$$
\left(\widetilde{M}_{d}\right)^{b}=\left(\begin{array}{cc}
\widetilde{M}_{d-1} & \widetilde{B}_{d} \\
\widetilde{B}_{d}^{T} & \widetilde{C}_{d}^{b}
\end{array}\right),
$$

with $\widetilde{C}_{d}^{b} \equiv \widetilde{W}^{T} \widetilde{M}_{d-1} \widetilde{W}=\left(K^{T}+L^{T} W^{T}\right)\left(J_{d-1}^{-1}\right)^{T}\left(J_{d-1}^{T} M_{d-1} J_{d-1}\right) J_{d-1}^{-1}(K+W L)=$ $(K+W L)^{T} M_{d-1}(K+W L)$. A block matrix calculation now shows that the last expression coincides with the $C$-block of $J^{T} M_{d}^{b} J$, so $\left(\widetilde{M}_{d}\right)^{b}$ coincides with $\widetilde{M_{d}^{b}} \equiv$ $J^{T} M_{d}^{b} J$. Now, if $C_{d}^{b}$ is Hankel in $M_{d}^{b}$, then $M_{d}^{b}$ is a moment matrix, and therefore so also is $\widetilde{M_{d}^{b}}$. Thus $\left(\widetilde{M}_{d}\right)^{b}\left(=\widetilde{M_{d}^{b}}\right)$ is a moment matrix, whence its $C_{d}^{b}$ block is Hankel. The converse follows by symmetry.

\section{Determining SEQUenCES FOR BIVARIATE POLYNOMIALS}

In this section we examine certain determining sequences for bivariate polynomials that will be used in proving Theorem[1.9, By a determining sequence for $\mathbb{R}[x, y]_{d}$ we mean a sequence of distinct points $\Gamma \equiv\left\{\left(x_{k}, y_{k}\right)\right\}_{k=1}^{\infty} \subset \mathbb{R}^{2}$ with the following property: for $p \in \mathbb{R}[x, y]_{d}$, if there exists $k_{p} \in \mathbb{N}$ such that $p\left(x_{k}, y_{k}\right)=0$ for every $k \geq k_{p}$, then $p \equiv 0$. In particular, $\Gamma$ is determining if each nonzero polynomial in $\mathbb{R}[x, y]_{d}$ has at most a finite number of zeros in $\Gamma$.

The following result will prove useful in relating the geometry of the support of a representing measure to the rank of a moment matrix.

Proposition 3.1. Suppose $\mathcal{P} \equiv\left\{p_{1}(x, y), \ldots, p_{t}(x, y)\right\}$ is a set of independent polynomials in $\mathbb{R}[x, y]_{d}$. If $w^{(j)} \equiv\left\{w_{i}^{(j)}\right\}_{i=1}^{\infty}$ is a determining sequence for $\mathbb{R}[x, y]_{d}$ $(1 \leq j \leq t)$, then given $k>0$, there exist integers $k_{1}, \ldots, k_{t} \geq k$ such that $V\left[\mathcal{P} ; w_{k_{1}}^{(1)}, \ldots, w_{k_{t}}^{(t)}\right]$ is invertible.

Note that we are not assuming that the determining sequences in Proposition 3.1 are distinct; however, in the proof of Theorem 1.9 in Section 4, the sequences will be distinct. Furthermore, the integers $k_{1}, \ldots, k_{t}$ need not be distinct, but clearly the points $w_{k_{1}}^{(1)}, \ldots, w_{k_{t}}^{(t)}$ are necessarily distinct. We also note that although we have formulated Proposition 3.1 only for 2 variables, the concept of determining sequence and the proof that we present below are valid as well for $n$ variables. 
Proof of Proposition 3.1. The proof is by induction on $t \geq 1$. Since $p_{1}(x, y) \not \equiv 0$ and $w^{(1)}$ is a determining sequence, there exists $k_{1} \geq k$ such that $p_{1}\left(w_{k_{1}}^{(1)}\right)=$ $\frac{1}{k_{1}^{m}} Q_{d}^{(m)}\left(w_{1}\right) \neq 0$, so the result holds for $t=1$. Assume by induction that the result holds up to $t-1$. Since $\mathcal{P}_{t-1} \equiv\left\{p_{1}, \ldots, p_{t-1}\right\}$ is independent, there exist $k_{1}, \ldots, k_{t-1} \geq k$ such that $V_{t-1} \equiv V\left[\mathcal{P}_{t-1} ; w_{k_{1}}^{(1)}, \ldots, w_{k_{t-1}}^{(t-1)}\right]$ is invertible. Let $V(x, y)$ be defined by

$$
V(x, y):=\left(\begin{array}{cccc}
p_{1}\left(w_{k_{1}}^{(1)}\right) & \cdots & p_{t-1}\left(w_{k_{1}}^{(1)}\right) & p_{t}\left(w_{k_{1}}^{(1)}\right) \\
\vdots & \vdots & \vdots & \vdots \\
p_{1}\left(w_{k_{t-1}}^{(t-1)}\right) & \cdots & p_{t-1}\left(w_{k_{t-1}}^{(t-1)}\right) & p_{t}\left(w_{k_{t-1}}^{(t-1)}\right) \\
p_{1}(x, y) & \cdots & p_{t-1}(x, y) & p_{t}(x, y)
\end{array}\right),
$$

and set $p(x, y):=\operatorname{det} V(x, y)\left(\in \mathbb{R}[x]_{d}\right)$. Expanding $\operatorname{det} V(x, y)$ with respect to the bottom row, we have

$$
p(x, y)=D_{1} p_{1}(x, y)-D_{2} p_{2}(x, y)+\cdots+(-1)^{t+1} D_{t} p_{t}(x, y),
$$

where each $D_{i}$ is a minor of $p(x, y)$ and $D_{t}=\operatorname{det} V_{t-1}(\neq 0$, by induction). Since $\left\{p_{1}, \ldots, p_{t}\right\}$ is independent in $\mathbb{R}[x, y]_{d}$ and $D_{t} \neq 0$, then $p(x, y) \not \equiv 0$ in $\mathbb{R}[x, y]_{d}$. Now, since $\left\{w_{i}^{(t)}\right\}_{i=1}^{\infty}$ is a determining sequence, there exists $k_{t} \geq k$ such that $p\left(w_{k_{t}}^{(t)}\right) \neq 0$, whence $V\left[\mathcal{P} ; w_{k_{1}}^{(1)}, \ldots, w_{k_{t}}^{(t)}\right]$ is invertible.

We require a determining sequence for $\mathbb{R}[x, y]_{d}$ that is compatible with a moment matrix construction in the proof of Theorem 1.9] and to this end we will focus on the sequence $\Gamma \equiv\left\{\left(k+\frac{1}{k^{m}}, \alpha k\right)\right\}_{k=1}^{\infty}$, where $m \in \mathbb{N}$ satisfies $m \geq d$ and $\alpha \in \mathbb{R}$ is nonzero.

Proposition 3.2. $\Gamma$ is a determining sequence for $\mathbb{R}[x, y]_{d}$. If $p \in \mathbb{R}[x, y]_{d}$ and $p \not \equiv 0$, then $p$ has at most $m d+d$ zeros of the form $\left(k+\frac{1}{k^{m}}, \alpha k\right)$, where $k \in \mathbb{N}$.

Let $p \in \mathbb{R}[x, y]_{d}, p(x, y)=\sum_{i, j \geq 0, i+j \leq d} a_{i j} x^{i} y^{j}$. For $\alpha \neq 0$ and $m \in \mathbb{N}, m \geq d$, let $q_{d}^{(m)}(x):=p\left(x+\frac{1}{x^{m}}, \alpha x\right)$, and define $Q_{d}^{(m)} \in \mathbb{R}[x]_{m d+d}$ by

$$
Q_{d}^{(m)}(x):=x^{m d} q_{d}^{(m)}(x)=x^{m d} \sum_{i, j \geq 0, i+j \leq d} a_{i j}\left(x+\frac{1}{x^{m}}\right)^{i}(\alpha x)^{j} .
$$

It follows from (3.2) that to prove Proposition 3.2, it suffices to show that $Q_{d}^{(m)}(x) \not \equiv$ 0 ; i.e., $Q_{d}^{(m)}$ has some nonzero coefficient. The coefficients of $Q_{d}^{(m)}$ as a polynomial in $\mathbb{R}[x]_{m d+d}$ are not the coefficients $a_{i j}$ of $p(x, y)$, but, rather, certain linear combinations of the $a_{i j}$. To show that at least one such coefficient is nonzero, we require some auxiliary results. In the sequel we say that a power monomial $x^{k}$ appearing in $Q_{d}^{(m)}$ is affiliated with coefficient $a_{i j}$ of $p(x, y)$ if the formal expansion of (3.2) into a sum of monomials contains one or more terms of the form $\gamma a_{i j} x^{k}$, say $\gamma_{r} a_{i j} x^{k}(1 \leq r \leq s)$ for some $s \geq 1$, where $\gamma_{r} \equiv \alpha^{j} \delta_{r}$ is a nonzero absolute constant (i.e., independent of all $a_{u v}$ ); we sometimes write this as $x^{k} \leadsto a_{i j}$. It is clear from (3.2) that for fixed $i, j, k, \sum_{r=1}^{s} \gamma_{r} a_{i j} x^{k}$ is a term of the form $\Gamma a_{i j} x^{k}$, where $\Gamma=\alpha^{j} \beta$ and $\beta$ is some binomial coefficient. For 
our purposes, it is necessary to identify exactly which monomial powers $x^{k}$ appear in $Q_{d}^{(m)}$ and with which coefficients of $p(x, y)$ each monomial power is affiliated. For example, if $p(x, y)=a_{00}+a_{10} x+a_{01} y+a_{20} x^{2}+a_{11} x y+a_{02} y^{2}$, then $Q_{2}^{(m)}(x)=a_{00} x^{2 m}+a_{10} x^{2 m+1}+a_{10} x^{m}+a_{01} \alpha x^{2 m+1}+a_{20} x^{2 m+2}+2 a_{20} x^{2 m+1}+$ $a_{20}+a_{11} \alpha x^{2 m+2}+a_{11} \alpha x^{m+1}+a_{02} \alpha^{2} x^{2 m+2}$, so the sequences of coefficients with which each power monomial of $Q_{2}^{(m)}$ is affiliated are as follows:

$x^{0} \leadsto a_{20} ; x^{m} \leadsto a_{10} ; x^{m+1} \leadsto a_{20}, a_{11} ; x^{2 m} \leadsto a_{00} ; x^{2 m+1} \leadsto a_{10}, a_{01} ; x^{2 m+2} \leadsto$ $a_{20}, a_{11}, a_{02}$.

To identify the powers and affiliations in $Q_{d}^{(m)}$, consider the index set for the monomials in $p(x, y), \mathbb{Z}_{d}^{2}:=\{(i, j) \mid i, j \geq 0, i+j \leq d\}$, and for $m \geq d$, let $\mathcal{S}_{m, d}=$ $\{r m+s: r, m \in \mathbb{Z}, 0 \leq r \leq d, 0 \leq s \leq r\}$. The requirement $m \geq d$ is necessary to insure that $\mathcal{S}_{m, d}$ contains $\frac{(d+1)(d+2)}{2}$ distinct elements. It is now easy to check that the map $\phi \equiv \phi_{d}: \mathbb{Z}_{d}^{2} \longrightarrow \mathcal{S}_{m, d}$, defined by $\phi(i, j)=(d-i) m+j$ is a bijection, with inverse $\psi: \mathcal{S}_{m, d} \longrightarrow \mathbb{Z}_{d}^{2}$ given by $\psi(r m+s)=(d-r, s)$. (Indeed, if $(i, j) \in \mathbb{Z}_{d}^{2}$, then $r \equiv d-i$ and $s \equiv j$ satisfy $0 \leq r \leq d$ and $s=j \leq d-i=r$, so $\phi$ is well-defined, and similarly for $\psi$.) Note also that

$$
\phi_{d-1}(i, j)+m=\phi_{d}(i, j)
$$

Now let $r_{d}(x)=\sum_{i, j \geq 0, i+j \leq d-1} a_{i j}\left(x+\frac{1}{x^{m}}\right)^{i}(\alpha x)^{j}$ (which we can interpret as $q_{d-1}^{(m)}(x)$ relative to $\left.\sum_{i, j \geq 0, i+j \leq d-1} a_{i j} x^{i} y^{j}\right)$, and set $s_{d}(x)=\sum_{i, j \geq 0, i+j=d} a_{i j}\left(x+\frac{1}{x^{m}}\right)^{i}(\alpha x)^{j}$, so that $q_{d}^{(m)}(x)=r_{d}(x)+s_{d}(x)$. Thus, $Q_{d}^{(m)}(x)=x^{m}\left(x^{(d-1) m} r_{d}(x)\right)+x^{m d} s_{d}(x)$, or

$$
Q_{d}^{(m)}(x)=x^{m} Q_{d-1}^{(m)}(x)+x^{m d} s_{d}(x) .
$$

Lemma 3.3. Let $d \geq 1, m \geq d$. For $(i, j) \in \mathbb{Z}_{d}^{2}$, the power monomials $x^{k}$ in $Q_{d}^{(m)}$ that are affiliated with coefficient $a_{i j}$ of $p(x, y)$ are $x^{\phi(i, j)}, x^{\phi(i, j)+m+1}, \ldots$, $x^{\phi(i, j)+i(m+1)}$.

Proof. The proof is by induction on $d \geq 1$. For $d=1, m \geq 1, Q_{1}^{(m)}(x)=a_{00} x^{m}+$ $a_{10} x^{m+1}+a_{10} x^{0}+a_{01} \alpha x^{m+1}$. The unique power affiliated with $a_{00}$ is $x^{m}$, and $\phi(0,0)=m, i=0$. The powers affiliated with $a_{10}$ are $x^{0}$ and $x^{m+1}$, and $\phi(1,0)=0$, $i=1$. The unique power affiliated with $a_{01}$ is $x^{m+1}$, and $\phi(0,1)=m+1, i=0$.

Assume the result is true up to $d-1$ and consider $Q_{d}^{(m)}$. For $i, j \geq 0$ with $i+j \leq d-1$, it follows by induction that the powers affiliated with $a_{i j}$ in $Q_{d-1}^{(m)}$ are precisely $x^{\phi_{d-1}(i, j)}, x^{\phi_{d-1}(i, j)+m+1}, \ldots, x^{\phi_{d-1}(i, j)+i(m+1)}$. Thus, (3.4) implies that the powers affiliated with $a_{i j}$ in $Q_{d}^{(m)}$ are precisely $x^{\phi_{d-1}(i, j)+m}, x^{\phi_{d-1}(i, j)+2 m+1}$, $\ldots, x^{\phi_{d-1}(i, j)+(i+1) m+i}$, and (3.3) implies that these powers coincide with $x^{\phi_{d}(i, j)}$, $x^{\phi_{d}(i, j)+m+1}, \ldots, x^{\phi_{d}(i, j)+i(m+1)}$.

Now consider the case $i, j \geq 0, i+j=d$. It follows from (3.4) that the powers affiliated with $a_{i j}$ in $Q_{d}^{(m)}$ are those affiliated with $a_{i j}$ in the formal expansion of $x^{m d} s_{d}(x)$, i.e., those powers which appear in the complete expansion of $x^{m d}\left(x+\frac{1}{x^{m}}\right)^{i}(\alpha x)^{d-i}$. The monomial powers in this expansion are $x^{m d} x^{i} x^{d-i}$, $x^{m d} x^{i-1} x^{-m} x^{d-i}, x^{m d} x^{i-2} x^{-2 m} x^{d-i}, \ldots, x^{m d} x^{-i m} x^{d-i}$, which simplify to $x^{m d+d}$, $x^{m d-m+d-1}, x^{m d-2 m+d-2}, \ldots, x^{m d-m i+d-i}$, and this sequence coincides with $x^{\phi(i, d-i)+i(m+1)}, x^{\phi(i, d-i)+(i-1)(m+1)}, \ldots, x^{\phi(i, d-i)}$. 
Corollary 3.4. For $(i, j) \in \mathbb{Z}_{d}^{2}$, the powers affiliated with $a_{i j}$ in $Q_{d}^{(m)}$ are $x^{\phi(i, j)}$, $x^{\phi(i-1, j+1)}, \ldots, x^{\phi(0, j+i)}$.

Proof. For $0 \leq a \leq i, \phi(i, j)+a(m+1)=(d-i) m+j+a m+a=(d-i+a) m+j+a=$ $(d-(i-a)) m+j+a=\phi(i-a, j+a)$. The result now follows from Lemma 3.3.

Note that every power of $x$ in the expansion of the right hand side of (3.2) is affiliated with some $a_{i j}$. The preceding result shows that each such power of $x$ is of the form $x^{\phi\left(i^{\prime}, j^{\prime}\right)}$ for some $\left(i^{\prime}, j^{\prime}\right) \in \mathbb{Z}_{d}^{2}$, and it also shows that every such power is affiliated with at least one coefficient in $Q_{d}^{(m)}$, namely, $a_{i^{\prime} j^{\prime}}$. Since $\phi: \mathbb{Z}_{d}^{2} \longrightarrow \mathcal{S}_{m, d}$ is a bijection, it also follows that the powers in $Q_{d}^{(m)}$ can be expressed as $x^{r m+s}$ with $0 \leq r \leq d, 0 \leq s \leq r$.

Proof of Proposition 3.2. It suffices to show that $Q_{d}^{(m)}$ has at most $m d+m$ distinct real roots. Suppose to the contrary that $Q_{d}^{(m)}$ has more than $m d+d$ roots. Since $\operatorname{deg} Q_{d}^{(m)} \leq m d+d$, then $Q_{d}^{(m)} \equiv 0$ in $\mathbb{R}[x]$, and we will show that this implies $p(x, y) \equiv 0$ in $\mathbb{R}[x, y]$, contradicting the hypothesis. We may list the powers corresponding to $\mathcal{S}_{m, d}$ by increasing degree as

$$
x^{0}, x^{m}, x^{m+1}, x^{2 m}, x^{2 m+1}, x^{2 m+2}, \ldots, x^{d m}, x^{d m+1}, \ldots, x^{d m+d} .
$$

We will prove by induction on the position, $\tau$, of a power in this list $(1 \leq \tau \leq$ $\left.\frac{(d+1)(d+2)}{2}\right)$ that for the $\tau$-th power $x^{r m+s}(0 \leq r \leq d, 0 \leq s \leq r)$, every coefficient $a_{i j}$ of $p(x, y)$ with which $x^{r m+s}$ is affiliated satisfies $a_{i j}=0$.

Note from Corollary 3.4 that if $x^{0}$ is affiliated with coefficient $a_{i j}$ of $p(x, y)$, then $0=\phi(i-\rho, j+\rho)$ for some $\rho, 0 \leq \rho \leq i$. Now $0=(d-(i-\rho)) m+j+\rho=$ $(d-i+\rho) m+j+\rho$, and thus $\rho=0, d=i, j=0$. Conversely, $\phi(d, 0)=0$, so $x^{0}$ is affiliated only with $a_{d, 0}$; since $Q_{d}^{(m)} \equiv 0$, it follows that $a_{d, 0}=0$.

Now assume by induction that for the first $\tau-1$ powers in (3.5), each coefficient $a_{i j}$ with which any of these powers is affiliated satisfies $a_{i j}=0$. Let $x^{r m+s}$ denote the $\tau$-th power (for some $r, s$ with $0 \leq r \leq d, 0 \leq s \leq r$ ). Now $r m+s=\phi(i, j)$ for a unique $(i, j) \in \mathbb{Z}_{d}^{2}$, and Corollary 3.4 shows that $x^{r m+s}$ is affiliated with $a_{i j}$. Next, suppose $x^{r m+s}$ is also affiliated with some other coefficient $a_{i^{\prime}, j^{\prime}},(i, j) \neq\left(i^{\prime}, j^{\prime}\right)$. Now $\phi\left(i^{\prime}, j^{\prime}\right)=r^{\prime} m+s^{\prime}$ for some $r^{\prime}, s^{\prime}$ with $0 \leq r^{\prime} \leq d, 0 \leq s^{\prime} \leq r^{\prime}$. Lemma 3.3 shows that $x^{\phi\left(i^{\prime}, j^{\prime}\right)}$ comes first in the sequence of powers affiliated with $a_{i^{\prime}, j^{\prime}}$, and since this sequence is ordered by strictly increasing degree of the powers, we have $r^{\prime} m+s^{\prime}<r m+s$. By induction, since $x^{r^{\prime} m+s^{\prime}}$ is a lower power than $x^{r m+s}$, it follows that $a_{i^{\prime}, j^{\prime}}=0$. Thus, whether or not $x^{r m+s}$ is affiliated only with $a_{i j}$, we can now represent the total coefficient of $x^{r m+s}$ in $Q_{d}^{(m)}$ as $\gamma a_{i j}$ for some nonzero absolute constant $\gamma$. Since $Q_{d}^{(m)} \equiv 0$, it now follows that $a_{i j}=0$, so every coefficient with which $x^{r m+s}$ is affiliated equals 0 . By induction, we conclude that for every power $x^{r m+s}(0 \leq r \leq d, 0 \leq s \leq r)$, each coefficient with which $x^{r m+s}$ is affiliated equals 0. Since, given $a_{i j}, x^{\phi(i, j)}$ is affiliated with $a_{i j}$ (Corollary 3.4), it follows that each $a_{i j}=0$. Since $p(x, y) \not \equiv 0$ by hypothesis, this contradiction implies that $Q_{d}^{(m)}$ has at most $m d+d$ real roots, which completes the proof.

The following example shows that if $m<d$, then we may have $p(x, y) \not \equiv 0$, but $Q_{d}^{(m)} \equiv 0$. Let $d=2, m=1$, and for $\alpha \neq 0$ and $a_{11} \neq 0$, set $a_{00}=-\alpha a_{11}$, $a_{02}=-\frac{a_{11}}{\alpha}, a_{01}=a_{10}=a_{20}=0$. Then $p(x, y)=-a_{11}\left(\alpha-x y+\frac{1}{\alpha} y^{2}\right) \not \equiv 0$, but 
$q_{2}^{(1)}(x) \equiv p\left(x+\frac{1}{x}, \alpha x\right)=-a_{11}\left(\alpha-\left(\alpha x^{2}+\alpha\right)+\frac{1}{\alpha}\left(\alpha^{2} x^{2}\right)\right)=0$. Thus $Q_{2}^{(1)}(x) \equiv$ $x q_{2}^{(1)}(x)=0$.

We continue this section by identifying another determining sequence that we will use in proving Theorem 1.9 .

Proposition 3.5. Let $m \in \mathbb{N}, m \geq d$. If $p \in \mathbb{R}[x, y]_{d}$ and $p \not \equiv 0$, then $p$ has at most $m d+d$ zeros of the form $\left(\frac{1}{k^{m}}, k\right)$, where $k \in \mathbb{N}$.

Proof. For $p(x, y) \equiv \sum_{i, j \geq 0, i+j \leq d} a_{i j} x^{i} y^{j}$, let $r_{d}^{(m)}(x):=p\left(\frac{1}{x^{m}}, x\right)$, and define $R_{d}^{(m)} \in$ $\mathbb{R}[x]_{m d+d}$ by

$$
R_{d}^{(m)}(x):=x^{m d} r_{d}^{(m)}(x)=x^{m d} \sum_{i, j \geq 0, i+j \leq d} a_{i j}\left(\frac{1}{x^{m}}\right)^{i} x^{j} .
$$

We will prove that

$$
R \equiv R_{d}(x)=\sum_{i=0}^{d} \sum_{j=0}^{i} a_{d-i, j} x^{i m+j} .
$$

If $p$ has more than $m d+m$ zeros of the form $\left(\frac{1}{k^{m}}, k\right)$, then $R$ has more than $m d+m$ roots, so $R \equiv 0$ in $\mathbb{R}[x]$. Since $m \geq d$, the powers $x^{i m+j}(0 \leq i \leq d$, $0 \leq j \leq i)$ are distinct; moreover, each coefficient of $p$ is of the form $a_{d-i, j}$ for some $i, j$ with $0 \leq i \leq d, 0 \leq j \leq i$. It thus follows from (3.7) that each coefficient of $p$ equals 0 , so $p \equiv 0$, a contradiction. It remains to establish (3.7). Write $p(x, y)=u(x, y)+v(x, y)$, where $u(x, y)=\sum_{i, j \geq 0, i+j \leq d-1} a_{i j} x^{i} y^{j}$ and $v(x, y)=$ $\sum_{i, j \geq 0, i+j=d} a_{i j} x^{i} y^{j}$. Then $R_{d}(x)=x^{m} x^{(d-1) m} u\left(\frac{1}{x^{m}}, x\right)+x^{m d} v\left(\frac{1}{x^{m}}, x\right)$, and thus

$$
R_{d}(x)=x^{m} R_{d-1}(x)+x^{m d} v\left(\frac{1}{x^{m}}, x\right) .
$$

Now (3.7) follows from (3.8) by a straightforward induction on $d \geq 1$; we omit the details.

We conclude this section by describing a determining sequence, corresponding to a prescribed point $\left(x_{0}, y_{0}\right) \in \mathbb{R}^{2}$, that we will use in Section 5 . For $r, s \in \mathbb{N}$, with $s>0$ and $r>(d-1) s$, consider the sequence $\Lambda:=\left\{\left(x_{0}+\frac{1}{k^{r}}, y_{0}+\frac{1}{k^{r+s}}\right)\right\}_{k=1}^{\infty}$.

Proposition 3.6. $\Lambda$ is a determining sequence for $\mathbb{R}[x, y]_{d}$. If $p \in \mathbb{R}[x, y]_{d}$ and $p \not \equiv 0$, then $p$ has at most $(r+s) d$ zeros of the form $\left(x_{0}+\frac{1}{k^{r}}, y_{0}+\frac{1}{k^{r+s}}\right)$, where $k \in \mathbb{N}$.

The hypothesis $s>0$ is necessary: for $d=1$, if $a, b, c \in \mathbb{R}$ are nonzero, with $b=-c$, if $a+b x_{0}+c y_{0}=0$, and if we take $r=1$ and $s=0$, then $p(x, y):=a+b x+c y$ vanishes on $\Lambda$. We will use notation similar to that in the proof of Proposition 3.2 . For $p \in \mathbb{R}[x, y]_{d}, p(x, y)=\sum_{i, j \geq 0, i+j \leq d} a_{i j} x^{i} y^{j}$, let $q(x)=p\left(x_{0}+\frac{1}{x^{r}}, y_{0}+\frac{1}{x^{r+s}}\right)$, and define $Q_{d} \in \mathbb{R}[x]_{(r+s) d}$ by

$$
Q_{d}(x):=x^{(r+s) d} q(x)=x^{(r+s) d} \sum_{i, j \geq 0, i+j \leq d} a_{i j}\left(x_{0}+\frac{1}{x^{r}}\right)^{i}\left(y_{0}+\frac{1}{x^{r+s}}\right)^{j} .
$$


It follows readily that

$$
Q_{d}=x^{r+s} Q_{d-1}+x^{(r+s) d} \sum_{i=0}^{d} a_{i, d-i}\left(x_{0}+\frac{1}{x^{r}}\right)^{i}\left(y_{0}+\frac{1}{x^{r+s}}\right)^{d-i} .
$$

Lemma 3.7. The powers that appear in $Q_{d}$ are $x^{i r+j s}$ with $0 \leq i \leq j \leq d$, and

$$
x^{i r+j s} \leadsto a_{j-i+a, d-j+b}(a, b \geq 0, a+b \leq i) .
$$

Moreover, the complete expansion of (3.10) includes the term $a_{j-i, d-j} x^{i r+j s}$ (corresponding to $a=b=0$ in (3.11) ).

We have already seen that $s>0$ is necessary for Proposition 3.6. In order for the exponents $i r+j s(0 \leq i \leq j \leq d)$ to be distinct, it is necessary and sufficient that $r>(d-1) s$, which is the requirement in the definition of $\Lambda$.

Proof of Lemma 3.7. The proof is by induction on $d \geq 1$. For $d=1, p(x, y)=$ $a_{00}+a_{10} x+a_{01} y$, so $Q_{1}(x)=a_{00} x^{r+s}+a_{10} x_{0} x^{r+s}+a_{10} x^{s}+a_{01} y_{0} x^{r+s}+a_{01}$. Thus $x^{0}$ is affiliated only with $a_{01}$ (in agreement with $i=j=0, a=b=0$ in (3.11)), and $x^{s}$ is affiliated only with $a_{10}$ (in agreement with $i=0, j=1, a=b=0$ ). For $x^{r+s}$, we have $i=j=1$, and this power is affiliated with $a_{00}(a=b=0), a_{01}(a=0$, $b=1)$, and $a_{10}(a=1, b=0)$. By inspection, we also see that in $Q_{1}(x)$ we have the required terms $a_{01} x^{0}, a_{10} x^{s}$, and $a_{00} x^{r+s}$.

Now assume the result holds for $d-1$. Thus, in $Q_{d-1}$, for $0 \leq i \leq j \leq d-1$, the power $x^{i r+j s}$ is affiliated precisely with the coefficients $a_{j-i+a,(d-1)-j+b}$, where $a, b \geq 0, a+b \leq i$; moreover, the expansion of (3.10) (with $d-1$ taking the place of $d$ ) contains the term $a_{j-i,(d-1)-j} x^{i r+j s}$. It follows from (3.10) that in $Q_{d}$ the power $x^{(i+1) r+(j+1) s}$ is also affiliated with these coefficients. Setting $i^{\prime}:=i+1$, $j^{\prime}:=j+1$, in $Q_{d}$, for $1 \leq i^{\prime} \leq j^{\prime} \leq d$, the power $x^{i^{\prime} r+j^{\prime} s}$ is affiliated with

$$
a_{j^{\prime}-i^{\prime}+a, d-j^{\prime}+b}\left(a, b \geq 0, a+b \leq i^{\prime}-1\right) ;
$$

moreover, the expansion of (3.10) for $Q_{d}$ contains the terms

$$
a_{j-i,(d-1)-j} x^{(i+1) r+(j+1) s}=a_{j^{\prime}-i^{\prime}, d-j^{\prime}} x^{i^{\prime} r+j^{\prime} s} .
$$

To complete the proof of (3.11) (but with $i$ and $j$ in (3.11) replaced by $i^{\prime}$ and $j^{\prime}$ ), it is necessary to show that i) for the case $i^{\prime}=0,0 \leq j^{\prime} \leq d, x^{j^{\prime} s} \leadsto a_{j^{\prime}, d-j^{\prime}}$ and $Q_{d}$ contains the term $a_{j^{\prime}, d-j^{\prime}} x^{j^{\prime} s}$; and ii) $x^{i^{\prime} r+j^{\prime} s}$ is affiliated with $a_{j^{\prime}-i^{\prime}+a, d-j^{\prime}+b}$ when $0 \leq i^{\prime} \leq j^{\prime} \leq d$ and $a+b=i^{\prime}$.

Note that in (3.10), the expansion of $x^{(r+s) d} \sum_{i=0}^{d} a_{i, d-i}\left(x_{0}+\frac{1}{x^{r}}\right)^{i}\left(y_{0}+\frac{1}{x^{r+s}}\right)^{d-i}$ is of the form

$$
x^{(r+s) d} \sum_{i=0}^{d} a_{i, d-i}\left(\sum_{u=0}^{i}\left(\begin{array}{l}
i \\
u
\end{array}\right) x_{0}^{u} x^{-r(i-u)}\right)\left(\sum_{v=0}^{d-i}\left(\begin{array}{c}
d-i \\
v
\end{array}\right) y_{0}^{v} x^{-(r+s)(d-i-v)}\right) .
$$

Upon further expansion, this shows that

$$
x^{(u+v) r+(v+i) s} \leadsto a_{i, d-i}(0 \leq i \leq d, 0 \leq u \leq i, 0 \leq v \leq d-i) .
$$

To show i), given $0 \leq j^{\prime} \leq d$, in (3.13) we let $i=j^{\prime}, u=v=0$ and see that $x^{i s}$ is affiliated with $a_{i, d-i}$. Further, with $u=v=0$, it is clear from (3.12) that $Q_{d}$ contains the term $a_{i, d-i} x^{i s}$. For ii), we have $1 \leq i^{\prime} \leq j^{\prime} \leq d, a+b=i^{\prime}$, and we seek to show that $x^{i^{\prime} r+j^{\prime} s} \leadsto a_{j^{\prime}-i^{\prime}+a, d-j^{\prime}+b}$. Set $\tilde{i} \equiv j^{\prime}-b$ so that $0 \leq \tilde{i} \leq d$ and 
$a_{j^{\prime}-i^{\prime}+a, d-j^{\prime}+b}=a_{\tilde{i}, d-\tilde{i}}$. Let $u:=i^{\prime}-b(0 \leq u \leq d)$ and $v:=b(0 \leq v \leq d-\tilde{i})$. It now follows from (3.13) that $x^{i^{\prime} r+j^{\prime} s}=x^{(u+v) r+(v+\tilde{i}) s} \leadsto a_{\tilde{i}, d-\tilde{i}}$, which proves ii) and completes the induction.

Proof of Proposition 3.6. If $p(x, y)$ has more than $(r+s) d$ zeros in $\Lambda$, then $Q_{d}$ has more than $(r+s) d$ distinct real roots, so $Q_{d} \equiv 0$. Under this assumption, we will prove that each coefficient of $p(x, y)$ equals 0 , in contradiction to $p \not \equiv 0$. From Lemma 3.7. with $i=0,0 \leq j \leq d, a=b=0$, we see that in $Q_{d}, x^{j s}$ is affiliated solely with $a_{j, d-j}$, so $a_{j, d-j}=0(0 \leq j \leq d)$. Let us assume by induction that for some $i, 1 \leq i \leq d$, we have $a_{u v}=0$ for $u, v \geq 0, d \geq u+v \geq d-i+1$. We will show that each coefficient of degree $d-i$ equals 0 . Lemma 3.7 implies that for $i \leq j \leq d$, the coefficient of $x^{i r+j s}$ in $Q_{d}$ is of the form

$\alpha_{00} a_{j-i, d-j}+\alpha_{10} a_{j-i+1, d-j}+\alpha_{01} a_{j-i, d-j+1}+\cdots+\alpha_{i 0} a_{j, d-j}+\cdots+\alpha_{0 i} a_{j-i, d-j+i}$ for certain absolute constants $\alpha_{u v}$ with $\alpha_{00}=1$. Except for $a_{j-i, d-j}$, each coefficient of $p(x, y)$ that appears in the preceding expression has degree at least $d-i+1$, so by induction each such coefficient equals 0 . It follows that the coefficient of $x^{i r+j s}$ in $Q_{d}$ reduces to $\alpha_{00} a_{j-i, d-j}$, whence $a_{j-i, d-j}=0$. Thus, each coefficient of $p$ of degree $d-i$ equals 0 ; the result now follows by induction.

\section{Limits of POSitiVe Flat MOMENT MATRiCES}

In this section we prove Theorem 1.9. Throughout this section $M_{d} \equiv M_{d}(\beta)$ (as in $(2.3))$ denotes a bivariate moment matrix of degree $2 d$. Now suppose $\beta^{(2 d-1)}$ has a $\kappa$-atomic representing measure $\mu$, so that $M_{d}[\mu]$ is of the form

$$
M_{d}[\mu]:=\left(\begin{array}{cc}
M_{d-1} & B_{d} \\
B_{d}^{T} & C_{d}[\mu]
\end{array}\right) .
$$

Let $\Delta \equiv \Delta[\mu]:=C_{d}-C_{d}[\mu], s \equiv \operatorname{rank} \Delta[\mu], \rho[\mu] \equiv s+\kappa$, and

$$
M_{\Delta}:=\left(\begin{array}{cc}
0 & 0 \\
0 & \Delta
\end{array}\right) .
$$

We now re-state Theorem 1.9 for ease of reference.

Theorem 4.1. Let $n=2, d \geq 1$. Suppose that $M_{d}(\beta) \succeq 0$ and that $\beta^{(2 d-1)}$ has a $\kappa$-atomic representing measure $\mu$. If $\Delta \equiv \Delta[\mu] \succeq 0$ and $\rho[\mu] \leq \rho_{d-1}$, then $M_{d} \in \overline{\mathcal{F}_{d}}$. Moreover, if $\rho[\mu] \leq \tau \leq \rho_{d-1}$, then there exists a sequence of positive flat moment matrices, $\left\{M_{d}^{(k)}\right\}$, such that $M_{d}(\beta)=\lim _{k \rightarrow \infty} M_{d}^{(k)}$ and for each $k$, rank $M_{d}^{(k)}=$ $\operatorname{rank} M_{d-1}^{(k)}=\tau$.

Proof. Denote $\mu$ as $\mu \equiv \sum_{i=1}^{\kappa} \alpha_{i} \delta_{w_{i}}$, where the points $w_{i}$ are distinct and each $\alpha_{i}>0$. If $\rho[\mu]<\rho_{d-1}$ and $\rho[\mu]<\tau \leq \rho_{d-1}$, choose additional points $w_{\rho[\mu]+1}, \ldots, w_{\tau}$ so that all of the $\tau-s$ points are distinct. (If $\tau=\rho[\mu]$, omit all reference to these additional points in the sequel.) Let $R, S \in \mathbb{N}$, with $S>0$ and $R>(d-1) S$. With $w_{j} \equiv\left(x_{j}, y_{j}\right)(1 \leq j \leq \kappa$ and $\kappa+s+1 \leq j \leq \tau)$, we apply Proposition 3.6 to define the determining sequences $w^{(j)} \equiv\left\{w_{k}^{(j)}\right\}_{k=1}^{\infty}(1 \leq j \leq \kappa$ and $\kappa+s+1 \leq j \leq \tau)$, where $w_{k}^{(j)}:=\left(x_{j}+\frac{1}{k^{R}}, y_{j}+\frac{1}{k^{R+S}}\right)$.

Next, note that $\Delta$ is a Hankel matrix, say $\Delta=\left(u_{i+j}\right)_{0 \leq i, j \leq d}$. We first consider the case when $\Delta$ is a moment matrix, i.e., $u_{0}>0$. If $\Delta \succ 0$ (positive definite), 
then $s=d+1$ and $\Delta$ has an $s$-atomic representing measure $\nu$ by Theorem 2.3 (cf. [CF1]). Otherwise, $\Delta$ is positive and singular, i.e., $s \leq d$, so [FN2, Theorem 3.1] implies that there is a sequence, $\left\{H_{d}\left(y^{[k]}\right)\right\}$, of positive Hankel matrices such that $\Delta=\lim _{k \rightarrow \infty} H_{d}\left(y^{[k]}\right)$ and $\operatorname{rank} H_{d}\left(y^{[k]}\right)=\operatorname{rank} H_{d-1}\left(y^{[k]}\right)=s$. It follows from Theorem 1.4 that $H_{d}\left(y^{[k]}\right)$ admits an $s$-atomic representing measure $\nu_{k}$. Thus, for the remainder of the proof of this case, replacing $\Delta$ by $H_{d}\left(y^{[k]}\right)$ and $C_{d}$ by $C_{d}[\mu]+H_{d}\left(y^{[k]}\right)$ if necessary, we may assume that $\operatorname{rank} \Delta=s$ and that $\Delta$ admits an $s$-atomic representing measure $\nu \equiv \sum_{i=\kappa+1}^{\kappa+s} a_{i} \delta_{\left\{\alpha_{i}\right\}}$ (where each $a_{i}>0$ and the $\alpha_{i}$ are distinct). Further, for the purposes of approximation, in the sequel we may assume that $\alpha_{i} \neq 0(\kappa+1 \leq i \leq \kappa+s)$. For if a (unique) $\alpha_{i^{\prime}}$ satisfies $\alpha_{i^{\prime}}=0$, then consider the measures $\nu^{(l)}(l \geq 1)$ obtained from $\nu$ by replacing $\alpha_{i^{\prime}}=0$ by $\alpha_{i^{\prime}}=\frac{1}{l}$ (with $l$ large enough so that the $\alpha_{i}$ remain distinct). Clearly, $\Delta=\lim _{l \rightarrow \infty} H_{d}\left[\nu^{(l)}\right]$, and we claim that rank $H_{d}\left[\nu^{(l)}\right]=s$ for all sufficiently large $l$. Since $\nu^{(l)}$ is $s$ atomic, then (2.5) implies that $\operatorname{rank} H_{d}\left[\nu^{(l)}\right] \leq s$. The reverse inequality follows from lower semicontinuity of rank (cf. Section 1), since $\Delta=\lim _{l \rightarrow \infty} H_{d}\left[\nu^{(l)}\right]$ and rank $\Delta=s$. In the sequel, by replacing $\Delta$ by $H_{d}\left[\nu^{(l)}\right]$ (for sufficiently large $l$ ), we may thus assume that $\operatorname{rank} \Delta=s$ and that $\Delta$ has an $s$-atomic measure $\nu$ (as above) in which the $\alpha_{i}$ are distinct and nonzero. Now, for $\kappa+1 \leq j \leq \kappa+s$, choose an integer $m_{j} \geq d-1$. Applying Proposition 3.2 with $d$ replaced by $d-1$, we define the determining sequences $w^{(j)} \equiv\left\{w_{k}^{(j)}\right\}_{k=1}^{\infty}(\kappa+1 \leq j \leq \kappa+s)$, where $w_{k}^{(j)}:=\left(k+\frac{1}{k^{m j}}, \alpha_{j} k\right)$.

Let $v \equiv \rho_{d-1}$ and $t \equiv \tau$. Let $p_{1}(x, y), \ldots, p_{v}(x, y)$ denote the basis for $\mathbb{R}[x, y]_{d-1}$ consisting of all of the monomials, and let $\mathcal{P}:=\left\{p_{1}, \ldots, p_{t}\right\}$. We next apply Proposition 3.1 to $\mathcal{P}$ and the determining sequences $w^{(j)}(1 \leq j \leq t)$. It follows that given $k>0$, there exist $k_{1}, \ldots, k_{t} \geq k$ such that $V \equiv V\left[\mathcal{P}, w_{k_{1}}^{(1)}, \ldots, w_{k_{t}}^{(t)}\right]$ (as defined in Section 2) is invertible; in particular, the points $w_{j} \equiv w_{k_{j}}^{(j)}(1 \leq j \leq t)$ are distinct.

We define three atomic measures that will be used in constructing the flat approximants to $M_{d}$. Let

$$
\begin{gathered}
\mu^{(k)}:=\sum_{j=1}^{\kappa} \alpha_{j} \delta_{w_{j}}=\sum_{j=1}^{\kappa} \alpha_{j} \delta_{\left(x_{j}+\frac{1}{k_{j}^{R}}, y_{j}+\frac{1}{k_{j}^{R+S}}\right)} \\
\nu^{(k)}:=\sum_{j=\kappa+1}^{\kappa+s} \frac{a_{j}}{k_{j}^{2 d}} \delta_{w_{j}} \equiv \sum_{j=\kappa+1}^{\kappa+s} \frac{a_{j}}{k_{j}^{2 d}} \delta_{\left\{\left(k_{j}+\frac{1}{k_{j}^{m}}, \alpha_{j} k_{j}\right)\right\}}, \\
\sigma^{(k)}:=\sum_{j=\kappa+s+1}^{t} \frac{1}{k} \delta_{w_{j}} \equiv \sum_{j=\kappa+s+1}^{t} \frac{1}{k} \delta_{\left(x_{j}+\frac{1}{k_{j}^{R}}, y_{j}+\frac{1}{k_{j}^{R+S}}\right)} .
\end{gathered}
$$

Now let $\omega^{(k)}:=\mu^{(k)}+\nu^{(k)}+\sigma^{(k)}$ and set

$$
M_{d}^{(k)} \equiv M_{d}\left[\omega^{(k)}\right]=M_{d}\left[\mu^{(k)}\right]+M_{d}\left[\nu^{(k)}\right]+M_{d}\left[\sigma^{(k)}\right] .
$$

Straightforward calculations show that $\lim _{k \rightarrow \infty} M_{d}\left[\mu^{(k)}\right]=M_{d}[\mu], \lim _{k \rightarrow \infty} M_{d}\left[\nu^{(k)}\right]=$ $M_{\Delta}$, and $\lim _{k \rightarrow \infty} M_{d}\left[\sigma^{(k)}\right]=0$. Thus, $\lim _{k \rightarrow \infty} M_{d}^{(k)}=M_{d}[\mu]+M_{\Delta}=M_{d}(\beta)$. 
To complete the proof of this case, it remains to show that $M_{d}^{(k)}$ is flat. Since $\omega^{(k)}$ is a representing measure for $M_{d}^{(k)}, \operatorname{supp} \omega^{(k)}=\left\{w_{1}, \ldots, w_{t}\right\}, \mathcal{P} \subset \mathbb{R}[x, y]_{d-1}$, and $V\left[\mathcal{P} ; w_{1}, \ldots, w_{t}\right]$ is invertible, the conclusion that $M_{d}^{(k)}$ is flat follows directly from Theorem 2.7. This completes the proof when $u_{0}>0$.

We next consider the case when $u_{0}=0$. In this case, it follows from Theorem 2.3 that $u_{j}=0(0 \leq j \leq 2 d-1)$ and $\gamma:=u_{2 d} \geq 0$. If $\gamma=0$, then $\Delta=0$ and $s=0$; in this case, we may proceed as above except that we skip the step involving $\Delta$ and $\nu^{(k)}$ and, in particular, we do not define the sequences $w^{(j)}(\kappa+1 \leq j \leq \kappa+s)$. Assuming that $\gamma>0$, we have $s=1$, and for fixed $m \geq d-1$, we use Proposition 3.5 to define the determining sequence $w^{(\kappa+1)} \equiv\left\{w_{k}^{(\kappa+1)}\right\}_{k=1}^{\infty}$, where $w_{k}^{(\kappa+1)}=\left(\frac{1}{k^{m}}, k\right)$. We next apply Proposition 3.1 exactly as before to produce the points $w_{1}, \ldots, w_{t}$ with an invertible Vandermonde $V$. We define $\mu^{(k)}$ and $\sigma^{(k)}$ as before, but we now define

$$
\nu^{(k)}:=\frac{\gamma}{k_{j}^{2 d}} \delta_{w_{k_{j}}^{(j)}}
$$

where $j=\kappa+1$. The proof now continues exactly as in the case $u_{0}>0$, beginning with the definition of $\omega^{(k)}:=\mu^{(k)}+\nu^{(k)}+\sigma^{(k)}$.

We illustrate Theorem 4.1, and particularly Corollary 1.11, with a continuation of Example 2.1.

Example 4.2. In Example 2.1 we have $M_{3} \succeq 0$ and $\operatorname{rank} M_{3}=6$, with column relations $X^{2}=1, X^{3}=X, X^{2} Y=Y$, and $Y^{3}=2 Y$. It is straightforward to check that by propagating these column relations forward, i.e., by defining $X^{4}:=X^{2}, X^{3} Y:=X Y, X^{2} Y^{2}:=Y^{2}, X Y^{3}:=2 X Y, Y^{4}:=2 Y^{2}$, we construct a positive flat moment matrix extension $M_{4}$. Thus $\beta$ has a 6 atomic representing measure $\mu$ which may be explicitly computed as described in Section 2 (cf. CF7]). The support of $\mu$ is the variety of $M_{4}$, which consists of the common solutions to $x^{2}=1$ and $y^{3}=2 y$, namely $\left(x_{1}, y_{1}\right) \equiv(-1,0),\left(x_{2}, y_{2}\right) \equiv(-1,-\sqrt{2})$, $\left(x_{3}, y_{3}\right) \equiv(-1, \sqrt{2}),\left(x_{4}, y_{4}\right) \equiv(1,0),\left(x_{5}, y_{5}\right) \equiv(1,-\sqrt{2}),\left(x_{6}, y_{6}\right) \equiv(1, \sqrt{2})$. Note that $\mathcal{B} \equiv\left\{1, X, Y, X Y, Y^{2}, X Y^{2}\right\}$ is a basis for the column space of $M_{3}$ and define corresponding polynomials $p_{1}(x, y):=1, p_{2}(x, y)=x, p_{3}(x, y):=y, p_{4}(x, y)=x y$, $p_{5}(x, y):=y^{2}, p_{6}(x, y)=x y^{2}$. The Vandermonde $V \equiv\left(p_{i}\left(x_{j}, y_{j}\right)\right)_{1 \leq i, j \leq 6}$ is invertible. Let $v$ be the vector of moments corresponding to the $p_{i}$, i.e., $v:=$ $\left(\beta_{00}, \beta_{10}, \beta_{01}, \beta_{11}, \beta_{02}, \beta_{12}\right)^{T}=(1,0,0,0,1,0)^{T}$. We compute $\alpha \equiv\left(\alpha_{1}, \alpha_{2}, \alpha_{3}, \alpha_{4}\right.$, $\left.\alpha_{5}, \alpha_{6}\right)^{T}:=V^{-1} v=\left(\frac{1}{4}, \frac{1}{8}, \frac{1}{8}, \frac{1}{4}, \frac{1}{8}, \frac{1}{8}\right)^{T}$. Then $\mu:=\sum_{i=1}^{6} \alpha_{i} \delta_{\left(x_{i}, y_{i}\right)}$ is a representing measure for $M_{3}$.

We now use the method of Theorem 4.1 to approximate $M_{3}$ with positive flat moment matrices. Using Proposition 3.6 with $d=3, r=3, s=1$ ( $r$ and $s$ here have the same meaning as in Proposition (3.6), we perturb the support of $\mu$ as follows. For $k>0,1 \leq j \leq 6$, let $x_{k}^{(j)}=x_{j}+\frac{1}{(j k)^{3}}$ and $y_{k}^{(j)}=y_{j}+\frac{1}{(j k)^{4}}$. We now define

$$
\omega^{(k)} \equiv \mu^{(k)}:=\sum_{j=1}^{6} \alpha_{j} \delta_{\left(x_{k}^{(j)}, y_{k}^{(j)}\right)} .
$$

Since $\Delta[\mu]=0$, we set $\nu^{(k)}=0$, and since $\rho[\mu]=6=\rho_{2}$, we set $\sigma^{(k)}=0$. With $M_{3}^{(k)}:=M_{3}\left[\mu^{(k)}\right]$, it is clear that $\lim _{k \rightarrow \infty} M_{3}^{(k)}=M_{3}$. We performed a numerical test 
using Mathematica, where all calculations are performed exactly in $\mathbb{Q}[\sqrt{2}]$. In particular, calculations of the ranks of the approximants are exact because there are no roundoff errors in the matrices. Let $\beta^{[k]}$ denote the moment sequence corresponding to $M_{3}^{(k)}$ and let $\Delta_{k}=\max _{a, b \geq 0, a+b \leq 6}\left|\beta_{a b}-\beta_{a b}^{[k]}\right|$. We found $\Delta_{1} \approx 0.4, \Delta_{2} \approx 0.09$, $\Delta_{3} \approx 0.05, \Delta_{4} \approx 0.02, \Delta_{5} \approx 0.01, \Delta_{10} \approx 0.002, \Delta_{20} \approx 0.0002, \Delta_{50} \approx 10^{-5}$. Perhaps surprisingly, every $M_{3}^{(k)}$ that we tested was flat, even for $k=1$, where the error is large. Note also that in implementing Proposition 3.1, for each $k>0$ we used $k_{j}=j k(1 \leq j \leq 6)$. Experiments with each $k_{j}=k$ did not yield flat approximants $M_{3}^{(k)}$.

We next illustrate Theorem 4.1 particularly Corollary 1.10, in a case where $C^{b}$ is Hankel. In such a case, let $\mu$ denote the unique, $\left(\operatorname{rank} M_{d-1}\right)$-atomic measure associated with $M^{b} \equiv M_{d}[\mu]$, let $\Delta \equiv \Delta[\mu]=\Delta^{b}$, and let $\kappa=r \equiv \operatorname{rank} M_{d-1}$. In any example of this type, we may apply the method of Theorem 4.1 directly or we may apply a slightly different and simpler approach that we next describe. In this approach, we do not define the determining sequences $w^{(1)}, \ldots, w^{(r)}$, and we replace $\mu^{(k)}$ with $\mu$. Further, if $r+s<\tau$, we choose $\alpha_{j}(r+s+1 \leq j \leq t)$ so that $\alpha_{1}, \ldots, \alpha_{t}$ are distinct and nonzero. For $m \geq d-1$, we use Proposition 3.2 to define determining sequences $w^{(j)} \equiv\left\{\left(k+\frac{1}{k^{m}}, \alpha_{j} k\right)\right\}_{k=1}^{\infty}(r+s+1 \leq j \leq t)$. Given $k \geq 1$, we then apply Proposition 3.1 to $p_{r+1}, \ldots, p_{t}$ and the sequences $w^{(r+1)}, \ldots, w^{(t)}$ to produce points $w_{r+1}, \ldots, w_{t}$ (dependent on $k$ ) leading to an invertible $(\tau-r) \times(\tau-r)$ Vandermonde $V$. As before, we use $w_{r+1}, \ldots, w_{r+s}$ to define $\nu^{(k)}$ and $w_{r+s+1}, \ldots, w_{t}$ to define $\sigma^{(k)}$. Setting $\omega^{(k)}:=\mu+\nu^{(k)}+\sigma^{(k)}$, the proof then proceeds along the lines of the proof of Theorem 4.1, we omit the details.

Example 4.3. To define

$$
M_{4} \equiv M_{4}\left(\beta^{(8)}\right)=\left(\begin{array}{cc}
M_{3} & B_{4} \\
B_{4}^{T} & C_{4}
\end{array}\right)
$$

we begin with

$$
M_{3}=\left(\begin{array}{cccccccccc}
1 & 0 & 1 & 1 & 0 & 2 & 0 & 2 & 0 & 5 \\
0 & 1 & 0 & 0 & 2 & 0 & 2 & 0 & 5 & 0 \\
1 & 0 & 2 & 2 & 0 & 5 & 0 & 5 & 0 & 14 \\
1 & 0 & 2 & 2 & 0 & 5 & 0 & 5 & 0 & 14 \\
0 & 2 & 0 & 0 & 5 & 0 & 5 & 0 & 14 & 0 \\
2 & 0 & 5 & 5 & 0 & 14 & 0 & 14 & 0 & 42 \\
0 & 2 & 0 & 0 & 5 & 0 & 5 & 0 & 14 & 0 \\
2 & 0 & 5 & 5 & 0 & 14 & 0 & 14 & 0 & 42 \\
0 & 5 & 0 & 0 & 14 & 0 & 14 & 0 & 42 & 0 \\
5 & 0 & 14 & 14 & 0 & 42 & 0 & 42 & 0 & 132
\end{array}\right) .
$$

$M_{3}$ is positive semidefinite, with $\operatorname{rank} M_{3}=7$, and column dependence relations $X^{2}=Y, X^{3}=X Y$, and $X^{2} Y=Y^{2}$. From Theorem 2.2, a positive $M_{4}$ requires that in $\left(\begin{array}{ll}M_{3} & B_{4}\end{array}\right)$ we have $X^{4}=X^{2} Y, X^{3} Y=X Y^{2}, X^{2} Y^{2}=Y^{3}$, so $B_{4}$ must 
be of the form

$$
B_{4}=\left(\begin{array}{ccccc}
2 & 0 & 5 & 0 & 14 \\
0 & 5 & 0 & 14 & 0 \\
5 & 0 & 14 & 0 & 42 \\
5 & 0 & 14 & 0 & 42 \\
0 & 14 & 0 & 42 & 0 \\
14 & 0 & 42 & 0 & 132 \\
0 & 14 & 0 & 42 & 0 \\
14 & 0 & 42 & 0 & 132 \\
0 & 42 & 0 & 132 & x \\
42 & 0 & 132 & x & y
\end{array}\right) .
$$

A calculation shows that there exists $W$ satisfying $B_{4}=M_{3} W$ and

$$
C^{b}=\left(\begin{array}{ccccc}
14 & 0 & 42 & 0 & 132 \\
0 & 14 & 0 & 132 & x \\
42 & 0 & 132 & x & y \\
0 & 132 & x & x^{2}+428 & x(y-416) \\
132 & x & y & x(y-416) & 179464+x^{2}-844 y+y^{2}
\end{array}\right) .
$$

In the sequel, to satisfy the requirement that $C^{b}$ be Hankel, we set $y=x^{2}+428$. We next define $C_{4}$ by

$$
C_{4}=\left(\begin{array}{ccccc}
15 & 0 & 43 & 0 & 133 \\
0 & 43 & 0 & 133 & x \\
43 & 0 & 133 & x & x^{2}+429 \\
0 & 133 & x & x^{2}+429 & x\left(x^{2}+12\right) \\
133 & x & x^{2}+429 & x\left(x^{2}+12\right) & x^{4}+13 x^{2}+1417+\epsilon
\end{array}\right)(\epsilon \geq 0)
$$

so that

$$
\Delta \equiv C_{4}-C^{b}=\left(\begin{array}{ccccc}
1 & 0 & 1 & 0 & 1 \\
0 & 1 & 0 & 1 & 0 \\
1 & 0 & 1 & 0 & 1 \\
0 & 1 & 0 & 1 & 0 \\
1 & 0 & 1 & 0 & 1+\epsilon
\end{array}\right)
$$

Since $\Delta \succeq 0$, then $M_{4} \succeq 0$. However, since $X^{4} \neq X^{2} Y$ in the column space, $M_{4}$ is not recursively generated and thus has no representing measure. Since $M_{4} \succeq 0$, we have $\operatorname{rank} M_{4}=\operatorname{rank} M_{3}+\operatorname{rank} \Delta$. Thus, $\operatorname{rank} M_{4}=9$ if $\epsilon=0$ and $\operatorname{rank} M_{4}=10$ if $\epsilon>0$, so in either case $M_{4}$ satisfies the hypotheses of Theorem 4.1, and we will illustrate how to approximate $M_{4}$ with positive flat moment matrices.

We begin with the case when $\epsilon=0$ and $\operatorname{rank} M_{4}=9$, and we let $\mu$ denote the unique (7-atomic) representing measure for $M^{b}$ (which can be explicitly computed from [CF7] cf. Section 2). We first choose $\tau=9$, i.e., we will approximate $M_{4}$ by flat moment matrices $M_{4}^{(k)}$ with $\operatorname{rank} M_{4}^{(k)}=9$. Since $\epsilon=0, \Delta$ has the unique representing measure $\nu:=\frac{1}{2} \delta_{\{1\}}+\frac{1}{2} \delta_{\{-1\}}$. Following the proof of Theorem 4.1 and the modified approach sketched above (in particular, applying Propositions 3.1 and 3.2), we choose $m=3$, and for $k \geq 1$, we set $k_{1}=k_{2}=k, a_{1}=a_{2}=\frac{1}{2}$, and $\alpha_{1}=1$, $\alpha_{2}=-1$. Now we define

$$
\nu^{(k)}:=\frac{1}{2 k^{8}} \delta_{\left(k+\frac{1}{k^{3}}, k\right)}+\frac{1}{2 k^{8}} \delta_{\left(k+\frac{1}{k^{3}},-k\right)} .
$$


Let $\omega^{(k)}:=\mu+\nu^{(k)}$ and set $M_{4}^{(k)}=M_{4}\left[\omega^{(k)}\right]$. Clearly, $\lim _{k \rightarrow \infty} M_{4}^{(k)}=M^{b}+M_{\Delta}=M_{4}$, and a symbolic calculation shows that $\operatorname{rank} M_{4}^{(k)}=\operatorname{rank} M_{3}^{(k)}=9$.

We next choose $\tau=10$. Note that for $k \geq 1$, Propositions 3.1 and 3.2 guarantee the existence of $k_{1}, k_{2}, k_{3} \geq k$ corresponding to an invertible Vandermonde, but there is no explicit formula for the $k_{i}$ values. In this case, if we choose $k_{1}=k_{2}=$ $k_{3}=k$, then we find $\operatorname{rank} M_{3}^{(k)}=9$, so $M_{4}^{(k)}$ (which has rank 10) is not flat. Instead, we define $\nu^{(k)}$ as above, except that we now try $k_{1}=k$ and $k_{2}=2 k$. Further, following the method discussed above, we try $k_{3}=3 k, \alpha_{3}=2$, and we define $\sigma^{(k)}:=\frac{1}{k^{10}} \delta_{\left\{\left(3 k+\frac{1}{(3 k)^{3}}, 6 k\right)\right\}}$. Setting $\omega^{(k)}:=\mu+\nu^{(k)}+\sigma^{(k)}$ and $M_{4}^{(k)}:=$ $M_{4}\left[\omega^{(k)}\right]$, we see that $\lim _{k \rightarrow \infty} M_{4}^{(k)}=M^{b}+M_{\Delta}+0=M_{4}$, and a symbolic calculation shows that $\operatorname{rank} M_{4}^{(k)}=\operatorname{rank} M_{3}^{(k)}=10$.

We next consider the case when $\epsilon>0$, i.e., rank $\Delta=3$. In this case, $\Delta$ is not recursively generated and so has no representing measure. Nevertheless, FN2 implies that $\Delta$ may be approximated by rank 3 positive flat Hankel matrices. Indeed, for $k>0$, if we define

$$
\nu_{k}:=\frac{1}{2} \delta_{\{1\}}+\frac{1}{2} \delta_{\{-1\}}+\frac{\epsilon}{k^{8}} \delta_{\{k\}},
$$

then $\lim _{k \rightarrow \infty} H_{4}\left[\nu_{k}\right]=\Delta$, and rank $H_{4}\left[\nu_{k}\right]=\operatorname{rank} H_{3}\left[\nu_{k}\right]=3$. We may therefore assume that $\Delta=\Delta^{(k)}:=H_{4}\left[\nu_{k}\right]$, and we proceed as outlined above, with $r=7$ and $\rho=\tau=\rho_{3}=10$. Let $\alpha_{1}=1, \alpha_{2}=-1, \alpha_{3}=k$, and $a_{1}=a_{2}=\frac{1}{2}, a_{3}=\frac{\epsilon}{k^{8}}$. For fixed $j \geq k$, we choose $k_{1}=j, k_{2}=2 j, k_{3}=3 j$ (based on Proposition 3.1 and experimentation), and define

$$
\nu^{(k)}=\frac{1}{2 k_{1}^{8}} \delta_{\left\{\left(k_{1}+\frac{1}{k_{1}^{3}}, k_{1}\right)\right\}}+\frac{1}{2 k_{2}^{8}} \delta_{\left\{\left(k_{2}+\frac{1}{k_{2}^{3}},-k_{2}\right)\right\}}+\frac{\epsilon}{k^{8}} \frac{1}{k_{3}^{8}} \delta_{\left\{\left(k_{3}+\frac{1}{k_{3}^{3}}, k \cdot k_{3}\right)\right\}} .
$$

We now set $\omega^{(k)}:=\mu+\nu^{(k)}$ and $M_{4}^{(k)}:=M_{4}\left[\omega^{(k)}\right]$. Clearly, $\lim _{k \rightarrow \infty} M_{4}^{(k)}=M^{b}+$ $\lim _{k \rightarrow \infty} M_{\Delta(k)}=M^{b}+M_{\Delta}=M_{4}$, and a symbolic calculation shows that $\operatorname{rank} M_{4}^{(k)}=$ $\operatorname{rank} M_{3}^{(k)}=10$.

To conclude the example we re-define $C_{4}$ as

$$
C_{4}=\left(\begin{array}{ccccc}
14 & 0 & 42 & 0 & 132 \\
0 & 42 & 0 & 132 & x \\
42 & 0 & 132 & x & x^{2}+428 \\
0 & 132 & x & x^{2}+428 & x\left(x^{2}+12\right) \\
132 & x & x^{2}+428 & x\left(x^{2}+12\right) & x^{4}+13 x^{2}+1416+\epsilon
\end{array}\right)(\epsilon>0)
$$

so that the Hankel matrix $\Delta$ is no longer a moment matrix, i.e., $\Delta_{j}=0(0 \leq j \leq 7)$ and $\Delta_{8}=\epsilon>0$. We have $r=7, \rho=8$, and we choose $\tau=9$. Using Propositions 3.1 and 3.5 (with $k_{1}=k$ and $k_{2}=2 k$ in Proposition 3.1), let $\nu^{(k)}:=\frac{\epsilon}{k^{8}} \delta_{\left\{\left(\frac{1}{k^{3}}, k\right)\right\}}$ and $\sigma^{(k)}:=\frac{1}{k^{10}} \delta_{\left\{\left(\frac{1}{(2 k)^{3}}, 2 k\right)\right\}}$. Setting $\omega^{(k)}:=\mu+\nu^{(k)}+\sigma^{(k)}$ and $M_{4}^{(k)}:=M_{4}\left[\omega^{(k)}\right]$, we see that $\lim _{k \rightarrow \infty} M_{4}^{(k)}=M^{b}+M_{\Delta}+0=M_{4}$, and a symbolic calculation shows that $\operatorname{rank} M_{4}^{(k)}=\operatorname{rank} M_{3}^{(k)}=9$. 
We conclude this section with a proof of Proposition 1.12

Proof of Proposition 1.12. The proof consists of repeating the proof of Theorem 4.1 up to the point where we define $\omega^{(k)}:=\mu^{(k)}+\nu^{(k)}+\sigma^{(k)}$, set

$$
M_{d}^{(k)} \equiv M_{d}\left[\omega^{(k)}\right]=M_{d}\left[\mu^{(k)}\right]+M_{d}\left[\nu^{(k)}\right]+M_{d}\left[\sigma^{(k)}\right],
$$

and show that $\lim _{k \rightarrow \infty} M_{d}^{(k)}=M_{d}[\mu]+M_{\Delta}=M_{d}(\beta)$. This shows that $M_{d}$ is in the closure of moment matrices with measures, so $L_{\beta}$ is positive. Since we are not interested in the ranks of the approximating moment matrices, it is also possible to re-work the proof in a simpler way, without using determining sequences.

\section{Flat approximation in the Degree 6 moment PRoblem}

Our main application is Theorem 1.1, which is included in the following result.

Theorem 5.1. $M_{3} \equiv M_{3}\left(\beta^{(6)}\right)$ belongs to $\overline{\mathcal{F}_{3}}$ if and only if $M_{3} \succeq 0$ and $\rho \equiv$ rank $M_{3} \leq 6$. In this case, given $\tau, \rho \leq \tau \leq 6, M_{3}$ is in the closure of the rank- $\tau$ positive flat moment matrices, and there exists a sequence of (computable) $\tau$-atomic positive measures $\left\{\mu_{k}\right\}$ such that $\beta_{i j}=\lim _{k \rightarrow \infty} \int x^{i} y^{j} d \mu_{k}(i, j \geq 0, i+j \leq 6)$. Further, either $M_{3}^{b}$ is a moment matrix or $M_{3}$ admits a flat extension $M_{4}$, whence $\beta^{(5)}$ has a representing measure.

Let $M_{d}=\left(\begin{array}{cc}M_{d-1} & B_{d} \\ B_{d}^{T} & C_{d}\end{array}\right)$ denote a positive semidefinite moment matrix. We recall from Section 2 two basic properties that we will use repeatedly. It follows from [CF2, Proposition 3.9] that if $M_{d} \succeq 0$, then

$$
p \in \mathbb{R}[x]_{d-1}, p(X)=0 \text { in } \operatorname{Col} M_{d-1} \Longrightarrow p(X)=0 \text { in } \operatorname{Col} M_{d} .
$$

Further, Theorem 2.2 iii) implies that $\left(\begin{array}{ll}M_{d-1} & B_{d}\end{array}\right)$ is recursively generated, i.e.,

$p, q, p q \in \mathbb{R}[x]_{d}, p(X)=0$ in $\operatorname{Col}\left(M_{d-1} B_{d}\right) \Longrightarrow(p q)(X)=0$ in $\operatorname{Col}\left(M_{d-1} B_{d}\right)$.

If $M_{d} \succeq 0$, then $\operatorname{Ran} B_{d} \subseteq \operatorname{Ran} M_{d-1}$, so there exists $W$ such that $B_{d}=M_{d-1} W$. Let $C_{d}^{\mathrm{b}}:=B_{d}^{T} W=W^{T} M_{d-1} W$; note that $C_{d}^{b}=C_{d}^{\mathrm{b}} W^{T}$ and that $C_{d}^{\mathrm{b}}$ is independent of $W$ satisfying $B_{d}=M_{d-1} W$. Let $M_{d}^{b}:=\left(\begin{array}{cc}M_{d-1} & B_{d} \\ B_{d}^{T} & C_{d}^{b}\end{array}\right)$. The following property is useful in computing $M_{d}^{b}$ :

$$
p \in \mathbb{R}[x]_{d}, p(X)=0 \text { in } C o l\left(\begin{array}{ll}
M_{d-1} & B_{d}
\end{array}\right) \Longrightarrow p(X)=0 \text { in } \operatorname{Col} M_{d}^{b} .
$$

As discussed in Section 2, $\operatorname{rank} M_{d}^{b}=\operatorname{rank} M_{d-1}$. Thus, if $C_{d}^{b}$ is Hankel, then $M_{d}^{b}$ is a flat moment matrix extension of $M_{d-1}$ (using the data in $B_{d}$ ) and thus has a representing measure (cf. Theorem 1.4). Note that in the case when $d=3$, since $C_{3}^{b} \equiv\left(c_{i j}\right)_{1 \leq i, j \leq 4}$ is real symmetric, $C_{3}^{b}$ is Hankel if and only if $c_{31}=c_{22}, c_{41}=c_{32}$, and $c_{42}=c_{33}$.

We begin the proof of Theorem 5.1 with a series of preliminary results based on the value of $r \equiv \operatorname{rank} M_{2}$.

Proposition 5.2. If $M_{3} \succeq 0$, rank $M_{3} \leq 6$ and $r \equiv \operatorname{rank} M_{2} \leq 3$, then $C_{3}^{b}$ is Hankel. 
Proof. Suppose $r=1$. Then in $\operatorname{Col} M_{3}, X=\alpha 1, Y=\beta 1$ (for certain scalars $\alpha, \beta$ ), so (5.2) and (5.3) imply that in $\operatorname{Col} M_{3}^{b}, X^{i} Y^{j}=\alpha^{i} \beta^{j} 1$. Then for $i+j=k+l=3$ with $1 \leq i, l \leq 3,0 \leq j, k \leq 2,\left\langle X^{i} Y^{j}, X^{k} Y^{l}\right\rangle=\alpha^{i+k} \beta^{j+l}=\left\langle X^{i-1} Y^{j+1}, X^{k+1} Y^{l-1}\right\rangle$, so $C_{3}^{b}$ is Hankel.

Next, let $r=2$. If $\operatorname{rank} M_{1}=1$, then by recursiveness in $M_{2}$, it follows that $\operatorname{rank} M_{2}=1$, a contradiction. Thus $\operatorname{rank} M_{1}=\operatorname{rank} M_{2}=2$, so $M_{2}$ is flat. Thus $M_{2}$ has a unique flat extension $\widetilde{M}_{3}$. Since $\widetilde{M}_{3}$ is recursively generated, it follows readily from (5.2) that $B_{3}=\widetilde{B}_{3}$. Now (5.3) implies that $M_{3}^{\mathrm{b}}=\widetilde{M}_{3}$, so $C_{3}^{\mathrm{b}}=\widetilde{C}_{3}$, whence $C_{3}^{b}$ is Hankel.

Now suppose $r=3$. As above, if $\operatorname{rank} M_{1}=1$, then by recursiveness of $M_{2}$, rank $M_{2}=1$, a contradiction. Further, if rank $M_{1}=3$, then $M_{2}$ is flat, so we may proceed exactly as in the $r=2$ case (above) to conclude that $C_{3}^{b}$ is Hankel. We may thus assume that $\operatorname{rank} M_{1}=2$, and we first consider the case when $\{1, X\}$ is a column basis, with $Y=\alpha 1+\beta X$ for some $\alpha, \beta \in \mathbb{R}$. By recursiveness in $M_{2}$, $X Y=\alpha X+\beta X^{2}$ and $Y^{2}=\alpha Y+\beta X Y$, so $\left\{1, X, X^{2}\right\}$ is a basis for $C o l M_{2}$. From (5.2), in $\mathrm{Col} \mathrm{B}_{3}$ we have

$$
\begin{gathered}
X^{2} Y=\alpha X^{2}+\beta X^{3}, \\
X Y^{2}=\alpha X Y+\beta X^{2} Y, \\
Y^{3}=\alpha Y^{2}+\beta X Y^{2} .
\end{gathered}
$$

These relations and the value of $\beta_{50}$ completely determine the other moments of degree 5; thus from (5.4), $\beta_{41}=\alpha \beta_{40}+\beta \beta_{50}, \beta_{32}=\alpha \beta_{31}+\beta \beta_{41}, \beta_{23}=\alpha \beta_{22}+\beta \beta_{32}$; from (5.5), $\beta_{14}=\alpha \beta_{13}+\beta \beta_{23}$; and from (5.6), $\beta_{05}=\alpha \beta_{04}+\beta \beta_{14}$. With these values, a calculation shows that there exists $W$ such that $B_{3}=M_{2} W$, and a further calculation shows that $C_{3}^{b} \equiv W^{T} M_{2} W$ is Hankel. In the remaining case, a basis is $\{1, Y\}$, and the proof is entirely analogous.

We next analyze cases of Theorem 5.1 with $r=4$ or $r=5$. Since $M_{3} \succeq 0$, then $M_{2}$ is recursively generated, so in these cases we must have $\{1, X, Y\}$ independent in $\mathrm{Col} M_{2}$. Thus, in $\mathrm{Col} M_{2}$ there is a dependence relation of the form $p(X, Y)=0$, with $\operatorname{deg} p(x, y)=2$. Given a degree-one map $\mathcal{T}$, let $\widetilde{M}_{2}$ denote the moment matrix corresponding to $M_{2}$ under $\mathcal{T}$ (cf. Proposition 2.8), and let $q(\widetilde{x}, \widetilde{y})=p \circ \mathcal{T}^{-1}(\widetilde{x}, \widetilde{y})$ $\in \mathbb{R}[\widetilde{x}, \widetilde{y}]_{d}$; clearly $q(\widetilde{x}, \widetilde{y})=0$ if and only if $p(x, y)=0$. It is well known that corresponding to $p(x, y)$ there is a degree-one map $\mathcal{T}$ such that the variety $q(\widetilde{x}, \widetilde{y})=$ 0 is one of the following: $\widetilde{x} \widetilde{y}=0, \widetilde{x} \widetilde{y}=1, \widetilde{y}=\widetilde{x}^{2}, \widetilde{x}^{2}+\widetilde{y}^{2}=1, \widetilde{x}^{2}=1, \widetilde{x}^{2}=0$, $\widetilde{x}^{2}=-1, \widetilde{x}^{2}+\widetilde{y}^{2}=0, \widetilde{x}^{2}+\widetilde{y}^{2}=-1$ (cf. [SH] p. 405]). We note that column dependence relations corresponding to any of the last four cases cannot occur in $\widetilde{M}_{2}$ if $\widetilde{M}_{2} \succeq 0$ and $\operatorname{rank} \widetilde{M}_{2} \geq 4$. To see this, we may scale $\widetilde{\beta}$ so that $\widetilde{\beta}_{00}=1$, and we denote $\widetilde{M}_{2}$ as

$$
\widetilde{M}_{2} \equiv\left(\begin{array}{cccccc}
1 & a & b & c & d & e \\
a & c & d & f & g & h \\
b & d & e & g & h & k \\
c & f & g & p & q & w \\
d & g & h & q & w & s \\
e & h & k & w & s & t
\end{array}\right)
$$


If $\widetilde{X}^{2}=0$, then $c=f=g=p=q=w=0$; since $c=0$, positivity of $\widetilde{M}_{2}$ implies $a=d=h=0$, and, similarly, $w=0$ implies $s=0$. Thus, rank $\widetilde{M}_{2} \leq 3$, a contradiction. Similarly, if $\widetilde{X}^{2}+\widetilde{Y}^{2}=0$, then $c+e=0$ and $p+w=0$ imply $c=e=p=w=0$, and it follows as above (via positivity) that $\operatorname{rank} \widetilde{M}_{2} \leq 2$. If $\widetilde{X}^{2}=-1$, then $c=-1$, which violates positivity in $\widetilde{M}_{2}$; similarly, if $\widetilde{X}^{2}+\widetilde{Y}^{2}=-1$, then $c+e=-1$, which is impossible. Motivated by the preceding discussion, in the following sequence of results we assume that $M_{2}$ admits a column dependence relation corresponding to one of the following varieties: $x y=0, x y=1, x^{2}=y$, $x^{2}=1$, or $x^{2}+y^{2}=1$. In each case we will show that under the hypotheses of Theorem 5.1, $M_{3}$ satisfies the following property:

$$
C_{3}^{b} \text { is Hankel or } M_{3} \text { admits a flat extension } M_{4} \text {. }
$$

In view of Theorem 1.4 and Corollaries 1.10 and 1.11, it is clear that (5.8) implies the conclusions of Theorem 5.1, including the existence of a representing measure for $\beta^{(5)}$.

In the sequel, to simplify certain symbolic calculations, we always scale $\beta$ so that $\beta_{00}=1$ (without loss of generality). If $\mathcal{B}$ is a collection of columns of $M_{d}$ which forms a basis of the column space of $M_{d}$, we will denote by $\left[M_{d}\right]_{\mathcal{B}}$ the compression of $M_{d}$ to the rows and columns indexed by the elements of $\mathcal{B}$. Thus, if $M_{d} \succeq 0$, then $\left[M_{d}\right]_{\mathcal{B}} \succ 0$.

Proposition 5.3. Suppose $M_{3} \succeq 0$, with $\rho \leq 6$ and $r=4$. If $M_{2}$ has a column relation $X Y=0$, then $C_{3}^{b}$ is Hankel.

Proof. As noted above, $\{1, X, Y\}$ is independent. Suppose first that $C o l M_{2}$ has the basis $\mathcal{B} \equiv\left\{1, X, Y, X^{2}\right\}$. Writing $Y^{2}=c_{1} 1+c_{2} X+c_{3} Y+c_{4} X^{2}$, in $\left(\begin{array}{cc}M_{2} & B_{3}\end{array}\right)$ we have $X^{2} Y=0,0=X Y^{2}=c_{1} X+c_{2} X^{2}+c_{4} X^{3}$ and $Y^{3}=c_{1} Y+c_{3} Y^{2}$. Further, $M_{2}$ is of the form

$$
M_{2}=\left(\begin{array}{llllll}
1 & a & b & c & 0 & d \\
a & c & 0 & e & 0 & 0 \\
b & 0 & d & 0 & 0 & f \\
c & e & 0 & g & 0 & 0 \\
0 & 0 & 0 & 0 & 0 & 0 \\
d & 0 & f & 0 & 0 & h
\end{array}\right)
$$

with

$$
\begin{aligned}
& J \equiv\left[M_{2}\right]_{\mathcal{B}}=\left(\begin{array}{cccc}
1 & a & b & c \\
a & c & 0 & e \\
b & 0 & d & 0 \\
c & e & 0 & g
\end{array}\right) \succ 0, \\
& \left(\begin{array}{llll}
c_{1} & c_{2} & c_{3} & c_{4}
\end{array}\right)^{T}=J^{-1}\left(\begin{array}{llll}
d & 0 & f & 0
\end{array}\right)^{T}, h=\left(\begin{array}{llll}
d & 0 & f & 0
\end{array}\right)\left(\begin{array}{llll}
c_{1} & c_{2} & c_{3} & c_{4}
\end{array}\right)^{T} .
\end{aligned}
$$

From the above column relations, $B_{3}$ must be of the form

$$
B_{3}=\left(\begin{array}{cccc}
e & 0 & 0 & f \\
g & 0 & 0 & 0 \\
0 & 0 & 0 & h \\
t & 0 & 0 & 0 \\
0 & 0 & 0 & 0 \\
0 & 0 & 0 & u
\end{array}\right)
$$


for certain $t$ and $u$. Since $X^{3}$ in $B_{3}$ belongs to $\operatorname{Ran} M_{2}$, it follows that $X^{3}=$ $d_{1} 1+d_{2} X+d_{3} Y+d_{4} X^{2}$, with $\left(d_{1} d_{2} d_{3} d_{4}\right)=J^{-1}(e, g, 0, t)^{T}$. In particular, $\gamma:=\left\langle X^{3}, Y^{2}\right\rangle=0$, and a symbolic calculation shows that

$$
\gamma=(d, 0, f, 0)\left(d_{1} d_{2} d_{3} d_{4}\right)^{T}=\frac{\left(b f-d^{2}\right)\left(e^{3}-2 c e g+a g^{2}+\left(c^{2}-a c\right) t\right)}{\operatorname{det}(J)} .
$$

Further, since $Y^{3}=c_{1} Y+c_{3} Y^{2}$, it follows that $u=c_{1} f+c_{3} h$. Now let

$$
W=\left(\begin{array}{cccc}
d_{1} & 0 & 0 & 0 \\
d_{2} & 0 & 0 & 0 \\
d_{3} & 0 & 0 & c_{1} \\
d_{4} & 0 & 0 & 0 \\
0 & 0 & 0 & 0 \\
0 & 0 & 0 & c_{2}
\end{array}\right)
$$

Then $M_{2} W=B_{3}$, and in $C_{3}^{b} \equiv W^{T} M_{2} \equiv\left(c_{i j}\right)_{1 \leq i, j \leq 4}$, we have $c_{31}=0=c_{22}$ and $c_{42}=0=c_{32}$. Thus $C_{3}^{b}$ is Hankel if and only if $c_{41}=0\left(=c_{32}\right)$. A symbolic calculation shows that

$$
c_{41}=\frac{-\gamma \omega}{\operatorname{det}(J)}\left(\omega \equiv-b d e^{2}+\left(c^{3}-2 a c e+e^{2}\right) f+\left(b c d+a^{2} f-c f\right) g\right),
$$

and since $\gamma=0$, then $c_{41}=0$. Thus $C_{3}^{b}$ is Hankel, which completes the proof in this case.

Suppose next that $X Y=0$ and that $B \equiv\left\{1, X, Y, Y^{2}\right\}$ is a basis for $C o l M_{2}$. In view of the previous case, we may assume that $X^{2} \in\langle 1, X, Y\rangle$. Thus, $X^{2}=c_{1} 1+$

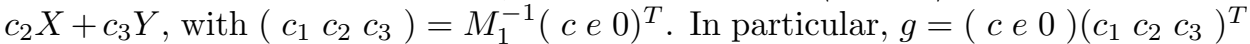
and $0=\left\langle X^{2}, Y^{2}\right\rangle=\left(\begin{array}{lll}d & 0 & f\end{array}\right)\left(\begin{array}{lll}c_{1} & c_{2} & c_{3}\end{array}\right)^{T}\left(\right.$ which entails $\left.\left.c^{2}-a e\right)\left(d^{2}-b f\right)=0\right)$. Denoting $M_{2}$ and $B_{3}$ as in (5.9) and (5.10), $t$ in $X^{3}$ is uniquely determined via recursiveness in $\left(M_{2} B_{3}\right)$ by

$$
X^{3}=c_{1} X+c_{2} X^{2}
$$

i.e., $t=c_{1} e+c_{2} g$.

Let $K=\left[M_{2}\right]_{\mathcal{B}}(\succ 0)$. Since $M_{3} \succeq 0, Y^{3}$ in $B_{3}$ is in $\operatorname{Ran} M_{2}$, so $Y^{3}=$ $k_{1} 1+k_{2} X+k_{3} Y+k_{4} Y^{2}$, where $\left(k_{1} k_{2} k_{3} k_{4}\right)=K^{-1}(f, 0, h, u)^{T}$. This relation and (5.10) imply that $0=\left\langle Y^{3}, X^{2}\right\rangle=k_{1} c+k_{2} e \equiv \lambda$. A symbolic calculation now shows that

$$
\lambda=\frac{\left(a e-c^{2}\right)\left(f^{3}-2 d f h+b h^{2}+\left(d^{2}-b f\right) u\right)}{\operatorname{det}(K)} .
$$

Setting

$$
W=\left(\begin{array}{cccc}
0 & 0 & 0 & k_{1} \\
c_{1} & 0 & 0 & k_{2} \\
0 & 0 & 0 & k_{3} \\
c_{2} & 0 & 0 & 0 \\
0 & 0 & 0 & 0 \\
0 & 0 & 0 & k_{4}
\end{array}\right),
$$

we have $M_{2} W=B_{3}$, and a further symbolic calculation shows that

$$
C_{3}^{b} \equiv B_{3}^{T} W=\left(\begin{array}{cccc}
* & 0 & 0 & c_{14} \\
0 & 0 & 0 & 0 \\
0 & 0 & 0 & 0 \\
0 & 0 & 0 & *
\end{array}\right),
$$


with

$$
c_{14} \equiv\left\langle Y^{3}, X^{3}\right\rangle=k_{1} e+k_{2} g=\frac{-\lambda\left(a c d+b^{2} e-d e\right)}{\operatorname{det}(K)} .
$$

Since $\lambda=0$, it follows that $C_{3}^{b}$ is Hankel, whence the result follows.

Proposition 5.4. Suppose $M_{3} \succeq 0$, with $\rho \leq 6$ and $r=4$. If $M_{2}$ has a column relation $X Y=1$, then $C_{3}^{b}$ is Hankel.

Proof. Since $X Y=1$, we may denote $M_{2}$ as

$$
M_{2}=\left(\begin{array}{llllll}
1 & a & b & c & 1 & d \\
a & c & 1 & e & a & b \\
b & 1 & d & a & b & f \\
c & e & a & g & c & 1 \\
1 & a & b & c & 1 & d \\
d & b & f & 1 & d & h
\end{array}\right)
$$

Since $r=4,\{1, X, Y\}$ is independent, and we first consider the case when $\mathcal{B} \equiv$ $\left\{1, X, Y, X^{2}\right\}$ is a column basis. Let

$$
J \equiv\left[M_{2}\right]_{\mathcal{B}}=\left(\begin{array}{cccc}
1 & a & b & c \\
a & c & 1 & e \\
b & 1 & d & a \\
c & e & a & g
\end{array}\right) \succ 0
$$

and set $\left(\begin{array}{llll}c_{1} & c_{2} & c_{3} & c_{4}\end{array}\right)^{T}:=J^{-1}\left(\begin{array}{llll}d & b & f & 1\end{array}\right)^{T}$. Then

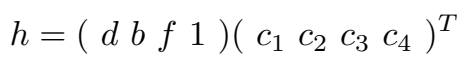

and $Y^{2}=c_{1} 1+c_{2} X+c_{3} Y+c_{4} X^{2}$. By recursiveness, in $C o l\left(M_{2} B_{3}\right)$ we have $X^{2} Y=X, X Y^{2}=Y, Y^{3}=c_{1} Y+c_{2} X Y+c_{3} Y^{2}+c_{4} X^{2} Y$. Now $B_{3}$ is of the form

$$
B_{3}=\left(\begin{array}{cccc}
e & a & b & f \\
g & c & 1 & d \\
c & 1 & d & h \\
t & e & a & b \\
e & a & b & f \\
a & b & f & u
\end{array}\right)
$$

for some $t \equiv \beta_{50}$ and $u \equiv \beta_{05}$. Setting $\left(k_{1} k_{2} k_{3} k_{4}\right)^{T}:=J^{-1}(\text { e g c } t)^{T}$, the condition $X^{3} \in \operatorname{Ran} M_{2}$, i.e., $X^{3}=k_{1} 1+k_{2} X+k_{3} Y+k_{4} X^{2}$, is equivalent to the condition that $\lambda:=k_{1} d+k_{2} b+k_{3} f+k_{4} 1-a$ satisfies $\lambda=0$. Setting

$$
W \equiv\left(\begin{array}{cccc}
k_{1} & 0 & 0 & 0 \\
k_{2} & 1 & 0 & c_{4} \\
k_{3} & 0 & 1 & c_{1} \\
k_{4} & 0 & 0 & 0 \\
0 & 0 & 0 & c_{2} \\
0 & 0 & 0 & c_{3}
\end{array}\right)
$$

we have $M_{2} W=B_{3}$, so that $C_{3}^{b}=B_{3}^{T} W$. Denoting $C_{3}^{b} \equiv\left(c_{i j}\right)_{1 \leq i, j \leq 4}$, a symbolic calculation shows that $c_{31}=c_{22}, c_{42}=c_{33}$, and $c_{32}=1$. Thus, $C_{3}^{b}$ is Hankel if and 
only if $c_{41} \equiv k_{1} f+k_{2} d+k_{3} h+k_{4} b$ satisfies $c_{41}=1\left(=c_{32}\right)$. Now, using (5.13), a symbolic calculation shows that

$$
c_{41}-1=\frac{\lambda \gamma}{\operatorname{det}(J)},
$$

where $\gamma$ is a polynomial in the moments of $M_{2}$. Since, from above, $\lambda=0$, we have $c_{41}=1$, whence $C_{3}^{b}$ is Hankel.

In the remaining case with $X Y=1$, we may assume that $M_{2}$ has the column basis $\mathcal{B} \equiv\left\{1, X, Y, Y^{2}\right\}$. In view of the previous case, we may also assume that

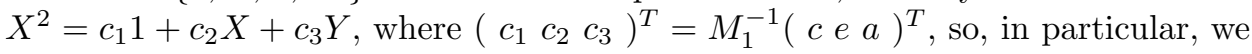
have

$$
c_{1} c+c_{2} e+c_{3} a=g, c_{1} d+c_{2} b+c_{3} f=1 .
$$

In $\left(M_{2} B_{3}\right)$, by recursiveness, we have $X^{3}=c_{1} X+c_{2} X^{2}+c_{3} 1, X^{2} Y=X$, $X Y^{2}=Y$. Further, since $Y^{3} \in \operatorname{Ran} M_{2}$, in $\operatorname{Col}\left(M_{2} B_{3}\right)$ we have

$$
Y^{3}=k_{1} 1+k_{2} X+k_{3} Y+k_{4} Y^{2},
$$

where $\left(\begin{array}{llll}k_{1} & k_{2} & k_{3} & k_{4}\end{array}\right)^{T}=L^{-1}\left(\begin{array}{lll}f d h u\end{array}\right)^{T}$ for

$$
L \equiv\left[M_{2}\right]_{\mathcal{B}}=\left(\begin{array}{cccc}
1 & a & b & d \\
a & c & 1 & b \\
b & 1 & d & f \\
d & b & f & h
\end{array}\right)(\succ 0)
$$

In particular, (5.15) entails

$$
b \equiv\left\langle Y^{3}, X^{2}\right\rangle=k_{1} c+k_{2} e+k_{3} a+k_{4} .
$$

Define $W$ by

$$
W=\left(\begin{array}{cccc}
c_{3} & 0 & 0 & k_{1} \\
c_{1} & 1 & 0 & k_{2} \\
0 & 0 & 1 & k_{3} \\
c_{2} & 0 & 0 & 0 \\
0 & 0 & 0 & 0 \\
0 & 0 & 0 & k_{4}
\end{array}\right),
$$

so that $M_{2} W=B_{3}$. A symbolic calculation shows that $C_{3}^{b}:=W^{T} M_{2} W \equiv$ $\left(c_{i j}\right)_{1 \leq i, j \leq 4}$ is Hankel if and only if $1=c_{41} \equiv\left\langle X^{3}, Y^{3}\right\rangle=c_{1} d+c_{2} b+c_{3} f$ and $c_{41}=c_{14}$; the former condition follows from (5.14) and the latter from the fact that $C_{3}^{b}$ is real symmetric, so the proof is complete.

Proposition 5.5. Suppose $M_{3} \succeq 0$, with $\rho \leq 6$ and $r=4$. If $M_{2}$ has a column relation $Y=X^{2}$, then $C_{3}^{b}$ is Hankel.

Proof. Since $r=4$ and $M_{2}$ is recursively generated, then $\{1, X, Y\}$ is independent, and we first consider the case when $\left\{1, X, Y, Y^{2}\right\}$ is a column basis for $M_{2}$. Thus, there is a column relation of the form $X Y=c_{1} 1+c_{2} X+c_{3} Y+c_{4} Y^{2}$. The corresponding curve $x y=c_{1}+c_{2} x+c_{3} y+c_{4} y^{2}$ has no $x^{2}$ term, so its discriminant is positive, and it thus represents a (possibly degenerate) hyperbola. Thus, by applying an appropriate degree-one map and Proposition 2.8 iii), iv), vii), $M_{2}$ may be transformed into a rank- 4 positive $\widetilde{M}_{2}$ with a column relation of the form $\widetilde{X} \widetilde{Y}=0$ or $\widetilde{X} \widetilde{Y}=\widetilde{1}$. It thus follows from Propositions 5.3 and 5.4 that the $C$ block of $\left(\widetilde{M}_{d}\right)^{b}$ is Hankel, whence Proposition $2.8 \mathrm{x}$ ) implies that $C_{3}^{b}$ is Hankel. 
We may now assume that $\{1, X, Y, X Y\}$ is a column basis, with a column relation of the form $Y^{2}=k_{1} 1+k_{2} X+k_{3} Y+k_{4} X Y$. Denoting $M_{2}$ as $M_{d}$ with $d=2$, we see that $M_{d}$ is recursively determinate in the sense of CF10; i.e., there is a degree-reducing relation $X^{n}=p(X, Y)(n=2$, deg $p=1<n)$ and a degreepreserving relation $Y^{m}=q(X, Y)(m=2$, deg $q=2=m)$. Since $M_{d}$ is positive, recursively generated, and recursively determinate with $n+m-2=d$, it follows from CF10, Corollary 2.4] that $M_{2}$ admits a unique flat extension $\widetilde{M}_{3}$. In this extension, $\widetilde{B}_{3}$ is completely determined from $M_{2}$ by recursiveness, i.e., $\widetilde{X^{3}}=\widetilde{X Y}$, $\widetilde{X^{2} Y}=\widetilde{Y^{2}}, \widetilde{X Y^{2}}=(x q)(\widetilde{X}, \widetilde{Y}), \widetilde{Y^{3}}=(y q)(\widetilde{X}, \widetilde{Y})$. Since $M_{2}=\widetilde{M_{2}}$ and $\left(M_{2} B_{3}\right)$ is recursively generated, the same column relations as just above determine $B_{3}$ so that $B_{3}=\widetilde{B}_{3}$. It now follows immediately from (5.3) that $C_{3}^{b}=\widetilde{C}_{3}$, and since $\widetilde{C}_{3}$ is Hankel, the result follows.

Proposition 5.6. Suppose $M_{3} \succeq 0$, with $\rho \leq 6$ and $r=4$. If $M_{2}$ has a column relation $X^{2}=1$, then $C_{3}^{b}$ is Hankel.

Proof. The proof is essentially the same as that of the preceding result. If $\{1, X$, $\left.Y, Y^{2}\right\}$ is a column basis for $M_{2}$, then there is a hyperbola relation $X Y=c_{1} 1+$ $c_{2} X+c_{3} Y+c_{4} Y^{2}$, so it follows as in the proof of Proposition 5.5 that $C_{3}^{b}$ is Hankel. In the remaining case, $\{1, X, Y, X Y\}$ is a column basis, and it follows exactly as in the proof of Proposition 5.5 that $M_{3}^{\mathrm{b}}$ is a moment matrix, whence $C_{3}^{\mathrm{b}}$ is Hankel.

Proposition 5.7. Suppose $M_{3} \succeq 0$, with $\rho \leq 6$ and $r=4$. If $M_{2}$ has a column relation $X^{2}+Y^{2}=1$, then $C_{3}^{b}$ is Hankel.

Proof. As in the previous proof, $\{1, X, Y\}$ is independent. We consider first the case when $\left\{1, X, Y, X^{2}\right\}$ is a column basis for $M_{2}$. It follows that $X Y$ is a linear combination of the basis columns, but since this relation has no $Y^{2}$ term, the relation represents a hyperbola, so the result follows from the hyperbola cases above (exactly as in the proof of Proposition 5.5, via Proposition 2.8 $\mathrm{x}$ )). In the remaining case, $\{1, X, Y\}$ is independent and $\left\{1, X, Y, X^{2}\right\}$ is dependent, so there is a degreereducing column relation of the form $X^{2}=c_{1} 1+c_{2} X+c_{3} Y$. Since we also have the degree-preserving relation $Y^{2}=1-X^{2}, M_{2}$ is recursively determinate, and the proof proceeds exactly as in the conclusion of the proof of Proposition 5.5 .

We next present cases where $r=5$. Since there is nothing to prove in Theorem 5.1 if $M_{3}$ is flat, and (5.8) clearly holds as well in this case, we may assume $\operatorname{rank} M_{3}=6$ in these cases.

Proposition 5.8. Suppose $M_{3} \succeq 0$, with $\rho=6$ and $r=5$. If $M_{2}$ has a column relation $X Y=0$, then $M_{3}$ satisfies (5.8).

Proof. We have $M_{2}$ as in (5.9), and $\left(M_{2} B_{3}\right)$ is recursively generated, so $B_{3}$ is as in (5.10). Since $X^{2} Y=X Y^{2}=0$ in $B_{3}$, (5.3) implies that the same column relations hold in $C_{3}^{b}$, so $C_{3}^{b}$ has the form

$$
C_{3}^{b}=\left(\begin{array}{cccc}
\kappa & 0 & 0 & \tau \\
0 & 0 & 0 & 0 \\
0 & 0 & 0 & 0 \\
\tau & 0 & 0 & \delta
\end{array}\right) .
$$


We may suppose $\tau \neq 0$, for otherwise $C_{3}^{b}$ is Hankel and the result follows. A symbolic calculation shows that $\tau=\tau_{1} \tau_{2}$ where

$$
\tau_{1}=\left(e^{3}-2 c e g+a g^{2}+\left(c^{2}-a e\right) t\right), \tau_{2}=\left(f^{3}-2 d f h+b h^{2}+\left(d^{2}-b f\right) u\right) .
$$

Let $J=\left[M_{2}\right]_{\mathcal{B}}$, where $\mathcal{B}$ denotes the column basis $\left\{1, X, Y, X^{2}, Y^{2}\right\}$. Now $J \succ 0$, so $\kappa=\left[X^{3}\right] J^{-1}\left[X^{3}\right]^{T}$, where $\left[X^{3}\right]=($ e g 0 t 0$)$; note that since $\tau \neq 0$ and $C_{3}^{b} \succeq 0$, then $\kappa>0$. Consider $\Delta:=C_{3}-C_{3}^{b}$ and write $\Delta \equiv\left(\Delta_{i j}\right)_{1 \leq i, j \leq 4}$.

We claim that $\Delta_{11} \neq 0$. Let us denote $C_{3}^{b}$ as $\left(c_{i j}\right)_{1 \leq i, j \leq 4}$, so that $c_{31}=c_{22}=$ $c_{13}=0$. Since $C_{3}\left(=C_{3}^{\mathrm{b}}+\Delta\right)$ is Hankel, it follows that $\Delta_{31}=\Delta_{22}=\Delta_{13}$. If $\Delta_{11}=0$, then, since $\Delta \succeq 0$, it follows that $\Delta_{21}=\Delta_{31}=\Delta_{41}=0$, whence $\Delta_{22}=0$. Positivity of $\Delta$ now implies $\Delta_{32}=\Delta_{42}=0$. Since $C_{3} \equiv C_{3}^{b}+\Delta$ is Hankel, it follows that $\Delta_{32}=\tau$, which contradicts $\tau \neq 0$.

Now $M_{3} \succeq 0$ and rank $M_{3}=6$, so

$$
\Delta \succeq 0, \operatorname{rank} \Delta=1 .
$$

Since $C_{3}^{b}+\Delta=C_{3}$ and $C_{3}$ is Hankel, (5.17) and (5.18) imply that for $p \equiv \Delta_{11}>0$ and certain scalars $q$ and $\epsilon, \Delta$ has the form

$$
\Delta=\left(\begin{array}{cccc}
p & q p & q^{2} p & \epsilon \\
q p & q^{2} p & q^{3} p & q \epsilon \\
q^{2} p & q^{3} p & q \epsilon & q^{2} \epsilon \\
\epsilon & q \epsilon & q^{2} \epsilon & k
\end{array}\right), \epsilon \neq q^{3} p .
$$

Since rank $\Delta=1, X Y^{2}=q^{2} X^{3}$ in $C o l \Delta$, whence $q^{4} p=q \epsilon$. Now, if $q \neq 0$, then $\epsilon=q^{3} p$, a contradiction. Thus $q=0$, so the Hankel matrix $C_{3}$ is of the form

$$
C_{3}=\left(\begin{array}{cccc}
v & 0 & 0 & 0 \\
0 & 0 & 0 & 0 \\
0 & 0 & 0 & 0 \\
0 & 0 & 0 & w
\end{array}\right)
$$

We now have $X^{2} Y=X Y^{2}=0$ in $M_{3}$, and we next construct a flat extension $M_{4}$. Since $\left(M_{3} B_{4}\right)$ must be recursively generated in any flat extension $M_{4}$, in $B_{4}$ we must define $X^{3} Y=X^{2} Y^{2}=X Y^{3}=0$. From this structure, it follows that in $B_{4}$,

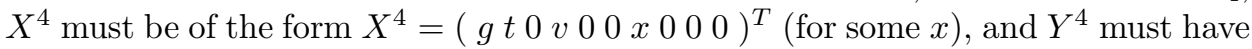
the form $Y^{4}=\left(\begin{array}{llllllll}h & 0 u & 0 & 0 & w & 0 & 0 & y\end{array}\right)^{T}$ (for some $y$ ). Since $p>0, M_{3}$ has a column basis $\left\{1, X, Y, X^{2}, Y^{2}, X^{3}\right\}$. Let $\widetilde{J}$ denote the compression of $M_{3}$ to these rows and columns, and let $\widetilde{X}^{4} \equiv(\operatorname{gt} 0 v 0 x)^{T}$. For each $x$, let $w_{x}=\widetilde{J}^{-1} \widetilde{X}^{4}$. For $M_{4}$ to be a positive extension of $M_{3}$ it is necessary that $X^{4} \in \operatorname{Ran} M_{3}$, and this is equivalent to the condition that $\psi \equiv\left\langle\widetilde{Y}^{3}, w_{x}\right\rangle=\left\langle X^{4}, Y^{3}\right\rangle=0$, where $\widetilde{Y}^{3} \equiv\left(\begin{array}{llllll}f & 0 & h & 0 & u & 0\end{array}\right)^{T}$. A symbolic calculation shows that

$$
\psi=\frac{\tau_{2}\left(Q-\tau_{1} x\right)}{\operatorname{det}(\widetilde{J})}
$$

(where $Q$ is some polynomial in the moments of $M_{3}$ ). Since $\tau_{1} \neq 0$, it follows that there exists a unique $x\left(=\frac{Q}{\tau_{1}}\right)$ such that $X^{4} \in \operatorname{Ran} M_{3}$. Further, setting $\tilde{Y}^{4}=\left(\begin{array}{llllll}h & 0 & u & 0 & w & 0\end{array}\right)^{T}$ and $w_{y}=\widetilde{J}^{-1} \widetilde{Y}^{4}$, if we define $y:=\left\langle\widetilde{Y}^{3}, w_{y}\right\rangle$, then the resulting $Y^{4}$ satisfies $Y^{4} \in \operatorname{Ran} M_{3}$, as required for positivity of $M_{4}$. With these values for the moments of $B_{4}$, we now compute $C_{4}^{b} \equiv\left(c_{i j}\right)_{1 \leq i, j \leq 5}$ via (5.3). Since $X^{3} Y=X^{2} Y^{2}=X Y^{2}=0$ in $C_{4}^{b}$, it is easy to see that $C_{4}^{b}$ is Hankel if and only if 
$c_{51}=0$. A symbolic calculation of $c_{51}$ using (5.3) shows that $c_{51}=\psi$, and by our choice of $x, \psi=0$, so the existence of a flat extension $M_{4}$ is established.

Proposition 5.9. Suppose $M_{3} \succeq 0$, with $\rho=6$ and $r=5$. If $M_{2}$ has a column relation $X Y=1$, then $M_{3}$ satisfies (5.8).

Proof. The proof is very similar to that of the previous result, so we omit many of the details. Symbolic calculation shows that either $C_{3}^{b}$ is Hankel, in which case the result follows, or $C_{3}^{b}$ is not Hankel because $\tau \equiv\left(C_{3}^{b}\right)_{41}-1 \neq 0$. In the latter case we proceed to construct a flat extension $M_{4}$ as follows. In $\left(M_{2} B_{3}\right)$ we have $X^{2} Y=X$ and $X Y^{2}=Y$. As in the previous proof, by examining the structures of $C_{3}^{b}$ and $\Delta$ in detail, we see that $X^{2} Y=X Y^{2}=0$ in $\Delta$, whence $X^{2} Y=X$ and $X Y^{2}=Y$ hold in $M_{3}$. In $M_{4}$ we must then have $X^{3} Y=X^{2}, X^{2} Y^{2}=1, X Y^{3}=Y^{2}$ in $\left(M_{3} B_{4}\right)$ (due to recursiveness), so in $B_{4}$ it remains to define $x \equiv \beta_{70}$ and $y \equiv \beta_{07}$. A symbolic calculation shows that there exists $x$ such that $X^{4} \in \operatorname{Ran} M_{3}$ if and only if an equation of the form $Q-\tau x=0$ has a solution (for a particular polynomial expression $Q$ in the moments of $M_{3}$ ). Since $\tau \neq 0$, there is a unique solution $x$. Next, $\beta_{07}$ can be defined so that $Y^{4} \in \operatorname{Ran} M_{3}$ exactly as in the proof of the preceding result. Now (5.3) implies that in $\operatorname{Col} C_{4}^{b}$ we have $X^{3} Y=X^{2}, X^{2} Y^{2}=1$, and $X Y^{3}=Y^{2}$, so $C_{4}^{b}$ is Hankel if and only if $\left(C_{4}^{b}\right)_{51}=1$. A symbolic calculation now shows that $\left(C_{4}^{b}\right)_{51}-1=(Q-\tau x) R$ (for some well-defined rational expression $R$ in the moment data). Since $Q-\tau x=0$, a flat extension $M_{4}$ is established, so the result follows.

Proposition 5.10. Suppose $M_{3} \succeq 0$, with $\rho=6$ and $r=5$. If $M_{2}$ has a column relation $Y=X^{2}$, then $M_{3}$ satisfies (5.8).

Proof. By recursiveness, we have $X^{3}=X Y$ and $X^{2} Y=Y^{2}$ in $\left(M_{2} B_{3}\right)$, and so, by (5.3), these relations also hold in $C_{3}^{b}$. Thus, $X^{3}$ and $X^{2} Y$ in $C_{3}^{b}$ are Hankel with respect to each other, and it follows that $C_{3}^{b} \equiv\left(c_{i j}\right)_{1 \leq i, j \leq 4}$ is Hankel if and only if $c_{42}=c_{33}$, in which case we are done. Assuming this is not the case, we will establish a flat extension $M_{4}$. Consider $\Delta:=C_{3}-C_{3}^{b}$, rank $\Delta=1$, and note that the leftmost two columns of $\Delta$ must be Hankel with respect to each other. If $p \equiv \Delta_{11} \neq 0$, then from the form of $C_{3}$ and $C_{3}^{b}$, it follows that $\Delta$ is of the form

$$
\Delta=\left(\begin{array}{cccc}
p & 0 & 0 & 0 \\
0 & 0 & 0 & 0 \\
0 & 0 & \delta & w \\
0 & 0 & w & s
\end{array}\right), \delta>0
$$

or

$$
\Delta=\left(\begin{array}{cccc}
p & q p & q^{2} p & q^{3} p \\
q p & q^{2} p & q^{3} p & q^{4} p \\
q^{2} p & q^{3} p & \delta & q^{5} p \\
q^{3} p & q^{4} p & q^{5} p & q^{6} p
\end{array}\right), \delta \neq q^{4} p, q \neq 0
$$

In the former case, rank $\Delta \geq 2$, a contradiction. In the latter case, since $p \neq 0$ and rank $\Delta=1$, then $\operatorname{Col} 3=q^{2} \operatorname{Col} 1$, which implies $\delta=q^{4} p$, a contradiction. Thus, $p=0$, so the positivity of $\Delta$ and the Hankel property of its leftmost two columns 
imply that

$$
\Delta=\left(\begin{array}{cccc}
0 & 0 & 0 & 0 \\
0 & 0 & 0 & 0 \\
0 & 0 & \delta & w \\
0 & 0 & w & s
\end{array}\right), \delta>0, \delta s=w^{2} .
$$

It follows that in $C_{3} \equiv C_{3}^{b}+\Delta$, we have $X^{3}=X Y$ and $X^{2} Y=Y^{2}$. Thus $M_{3}$ is positive and recursively generated. Since $\delta>0$, it follows that a column basis for $M_{3}$ is given by $\left\{1, X, Y, X Y, Y^{2}, X Y^{2}\right\}$. We thus have a column relation of the form $Y^{3}=k_{1} 1+k_{2} X+k_{3} Y+k_{4} X Y+k_{5} Y^{2}+k_{6} X Y^{2}$. Denoting $M_{3}$ as $M_{d}$ with $d=3$, we see that $M_{d}$ is recursively determinate, i.e., $X^{n}=p(X, Y)(n=2, \operatorname{deg} p=1)$ and $Y^{m}=q(X, Y)(m=3, \operatorname{deg} q=3)$. Since $M_{d}$ is positive, recursively generated, and recursively determinate with $n+m-2=d$, it follows from [CF10, Corollary 2.4] that $M_{3}$ admits a unique flat extension $M_{4}$, whence the result follows.

Proposition 5.11. Suppose $M_{3} \succeq 0$, with $\rho=6$ and $r=5$. If $M_{2}$ has a column relation $X^{2}=1$, then $M_{3}$ satisfies (5.8).

Proof. The proof is essentially the same as that of the preceding result; replacing $X^{2}=Y$ by $X^{2}=1$ has no effect on the argument there that either $C_{3}^{b}$ is Hankel or $M_{3}$ is recursively determinate with $n+m-2=d$ (in the sense of CF10, Corollary 2.4]), whence $M_{3}$ admits a flat extension $M_{4}$.

Proposition 5.12. Suppose $M_{3} \succeq 0$, with $\rho=6$ and $r=5$. If $M_{2}$ has a column relation $X^{2}+Y^{2}=1$, then $M_{3}$ admits a flat extension $M_{4}$.

Proof. Since $\{1, X, Y\}$ is independent in $\mathrm{Col} M_{3}$, it follows from [CF5, Proposition 1.12 ] that $M_{3}(\beta)$ corresponds to a complex moment matrix $\mathcal{M}_{3}(\gamma)$ with columns $1, Z, \bar{Z}$ independent and $\bar{Z} Z=1$ in the column space. [CF5, Theorem 1.1] now implies that $\mathcal{M}_{3}(\gamma)$ has a flat extension $\mathcal{M}_{4}$. Under the inverse correspondence of [CF5, Proposition 1.12], $\mathcal{M}_{4}$ corresponds to a real moment matrix $\widetilde{M}_{4}$ that is a flat extension of $M_{3}$.

We are now prepared to prove Theorem 5.1.

Proof of Theorem 5.1. The "only if" direction is clear from the discussion in Section 1. For the converse, let $r=\operatorname{rank} M_{2}$. If $r=6$, then $M_{3}$ is flat, so there is nothing to prove. For $1 \leq r \leq 3$, the result follows from Proposition 5.2 and Corollary 1.10. Suppose $4 \leq r \leq 5$. As noted earlier, in these cases recursiveness in $M_{2}$ implies that $\{1, X, Y\}$ is independent in $\mathrm{Col} M_{2}$. Since $M_{2}$ is singular, there is a column dependence relation of the form $A X^{2}+B X Y+C Y^{2}+D X+E Y+F 1=0$, with $A^{2}+B^{2}+C^{2} \neq 0$. Let $p(x, y)=A x^{2}+B x y+C y^{2}+D x+E y+F$ and consider the planar variety $p(x, y)=0$. As discussed earlier in this section (following Proposition 5.2), there is a degree-one map $\mathcal{T}(\mathcal{T}(x, y) \equiv(\widetilde{x}, \widetilde{y}))$, such that the variety corresponding to $q(\widetilde{x}, \widetilde{y}):=\left(p \circ \mathcal{T}^{-1}\right)(\widetilde{x}, \widetilde{y})$ is one of the following: $\widetilde{x} \widetilde{y}=0$, $\widetilde{x} \widetilde{y}=1, \widetilde{y}=\widetilde{x}^{2}, \widetilde{x}^{2}=1, \widetilde{x}^{2}+\widetilde{y}^{2}=1$. Applying $\mathcal{T}$ to $M_{3}$ via Proposition 2.8 it follows from Proposition $2.8 \mathrm{iii}$ ), iv), vii) that the resulting moment matrix $\widetilde{M}_{3}$ is positive, with $\operatorname{rank} \widetilde{M}_{3}=\operatorname{rank} M_{3},\{\tilde{1}, \tilde{X}, \widetilde{Y}\}$ independent in $\operatorname{Col} \widetilde{M}_{2}$, and with one of the following column dependence relations: $\widetilde{X Y}=\widetilde{0}, \widetilde{X Y}=\widetilde{1}, \widetilde{Y}=\widetilde{X^{2}}$, $\widetilde{X^{2}}=\widetilde{1}, \widetilde{X^{2}}+\widetilde{Y^{2}}=\widetilde{1}$. It now follows from Propositions 5.3-5.12 that $\widetilde{M}_{3}$ satisfies (5.8). Proposition $2.8 \mathrm{vi}$ ) and $\mathrm{x}$ ) thus imply that $M_{3}$ satisfies (5.8), so the proof is completed by applications of Corollaries 1.10 and 1.11 
The bivariate singular quartic problem was solved in CF5] (with one exception that we note below). We conclude with a new formulation based on Theorem 5.1

Proposition 5.13. For $n=2$, let $\beta \equiv \beta^{(4)}$ and suppose $M_{2}(\beta)$ is singular. The following are equivalent:

i) $\beta$ admits a representing measure;

ii) $M_{2}$ is positive and recursively generated, and rank $M_{2} \leq \operatorname{card} \mathcal{V}(\beta)$;

iii) $M_{2}$ admits a positive extension $M_{3} \equiv M_{3}(\widetilde{\beta})$ satisfying rank $M_{3} \leq 6$.

The equivalence of i) and ii) is proved in CF5, except that CF5 neglects to treat the case when $M_{2}$ has a column relation equivalent to $X^{2}=1$ under a degreeone map; we treat the $X^{2}=1$ case now.

Lemma 5.14. Suppose $X^{2}=1$ in Col $M_{2}$. Then $\beta \equiv \beta^{(4)}$ has a representing measure if and only if $M_{2}$ is positive, recursively generated, and satisfies rank $M_{2} \leq$ card $\mathcal{V}(\beta)$.

Proof. The necessity of the conditions is clear. For the converse, if $M_{1}$ is singular, the existence of a flat extension follows from [CF3] and from the equivalence of the real and complex truncated moment problems [CF7. We may thus assume that $M_{1} \succ 0$, and we consider next the case when $\{1, X, Y, X Y\}$ is a basis for $\operatorname{Col} M_{2}$. Then $Y^{2}=q(X, Y)$ for some $q \in \mathbb{R}[x, y]_{2}$, and since also $X^{2}=1$, it follows that $M_{2}$ is recursively determinate with $n+m-2=d$ (in the sense of [CF10, Corollary 2.4]), so CF10 implies the existence of a flat extension $M_{3}$. If $\left\{1, X, Y, Y^{2}\right\}$ is a column basis, then there is a hyperbola relation $X Y=a 1+b X+c Y+d Y^{2}$, so it follows from [CF8 that either $M_{2}$ has a flat extension $M_{3}$ or $M_{2}$ admits a positive, recursively generated extension of rank 5 , which in turn has a flat extension $M_{4}$.

In the remaining case, we have a column basis $\mathcal{B} \equiv\left\{1, X, Y, X Y, Y^{2}\right\}$, and we will construct a flat extension $M_{3}(\widetilde{\beta})$. Since $X^{2}=1$, we may denote $M_{2}$ by

$$
M_{2}=\left(\begin{array}{llllll}
1 & a & b & 1 & c & d \\
a & 1 & c & a & b & f \\
b & c & d & b & f & g \\
1 & a & b & 1 & c & d \\
c & b & f & c & d & p \\
d & f & g & d & p & q
\end{array}\right) .
$$

In $\operatorname{Col}\left(M_{2} B_{3}\right)$ we must have $X^{3}=X$ and $X^{2} Y=Y$, so $B_{3}$ has the form

$$
B_{3}=\left(\begin{array}{cccc}
a & b & f & g \\
1 & c & d & p \\
c & d & p & q \\
a & b & f & g \\
b & f & g & w \\
f & g & w & s
\end{array}\right)
$$

for certain $w, s$. Let $J=\left[M_{2}\right]_{\mathcal{B}}$. Since $J \succ 0$, it is clear that $B_{3}=M_{2} W$ for some $W$. Let $C_{3}^{b} \equiv W^{T} M_{2} W$. Since $X^{3}=X$ and $X^{2} Y=Y$ in $\operatorname{Col}\left(B_{3}^{T} C_{3}^{b}\right)$, then $C_{3}^{b} \equiv\left(c_{i j}\right)_{1 \leq i, j \leq 4}$ is Hankel if and only if $c_{42}=c_{33}$. A symbolic calculation shows that $\tau \equiv c_{42}-c_{33}$ is of the form $\tau=\frac{\tau_{1} \tau_{2}}{\text { Det } J}$, where $\tau_{1}$ and $\tau_{2}$ are polynomials in the moments of $M_{2}$. Further, $\tau_{1}$ can be expressed as $\tau_{1}=\sigma+\rho w$, where $\sigma$ and $\rho$ are also polynomials in the moments of $M_{2}$. Let $K$ denote the compression of $M_{2}$ to rows and columns indexed by $1, X, Y, X Y$. Then $K \succ 0$, and a symbolic 
calculation shows that $\operatorname{Det} K=\rho \rho^{\prime}$ for some moment polynomial $\rho^{\prime}$. Thus, $\rho \neq 0$, and it follows that there is a unique $w \equiv \widetilde{w}$ such that $\tau_{1}=0$. Setting $\widetilde{\beta}_{14}=\widetilde{w}$, then $\tau=0$ and $C_{3}^{b}$ is Hankel, so the existence of a flat extension $M_{3}(\widetilde{\beta})$ is established.

Proof of Proposition 5.13. We first show that ii) implies iii). If ii) holds and $M_{1}$ is singular, then CF3 and the equivalence of the real and complex truncated moment problems [CF7 together imply that $M_{2}$ has a flat extension, so iii) follows in this case. We now assume that $M_{1} \succ 0$, so that $M_{2}$ has a degree 2 column dependence relation. Note from Proposition 2.8 that all of the properties in i)-iii) (i.e., positivity, rank, variety, existence of measureness, recursiveness, existence of positive extensions with prescribed rank) are invariant under degree-one maps. From Proposition 2.8 and the discussion following Proposition 5.2. we may thus assume that $M_{2}$ has a column dependence corresponding to one of the five varieties considered in the proof of Theorem 5.1. In each of these cases, except for $x^{2}=1$, the results of [CF5] show that when ii) holds, then either $M_{2}$ admits a flat extension $M_{3}$ or $M_{2}$ has a positive extension $M_{3}$ with $\operatorname{rank} M_{3} \leq 1+\operatorname{rank} M_{2} \leq 6$, and $M_{3}$ admits a flat extension $M_{4}$. The proof of Lemma 5.14 establishes the same conclusions in the case when $x^{2}=1$. Thus, ii) implies iii). If iii) holds, then Theorem 5.1 implies that $L_{\widetilde{\beta}}$ is positive, so i) follows from Theorem 1.2. Since i) always implies ii) (cf. Section 2), the proof is complete.

\section{ACKNOWLEDGMENT}

The author wishes to thank Professor Jiawang Nie for an invitation to visit him at the University of California, San Diego, where this work commenced in January 2012. This paper is a sequel to [FN2], and the author benefited from helpful conversations with Professor Nie.

\section{ADDED IN PROOF}

A very general and concrete sufficient condition for positivity of $L_{\beta}$ was recently discovered by Grigoriy Blekherman [B]. Blekherman's results imply that if $n \geq 1$, $d \geq 3$, and $\operatorname{rank} M_{d} \leq 3 d-3$, then $L_{\beta}$ is positive. They also imply that Theorem 1.1 cannot be extended to moment problems of higher degree.

\section{REFERENCES}

[A] N. I. Akhiezer, The classical moment problem and some related questions in analysis, translated by N. Kemmer, Hafner Publishing Co., New York, 1965. MR0184042 (32 \#1518)

[BT] Christian Bayer and Josef Teichmann, The proof of Tchakaloff's theorem, Proc. Amer. Math. Soc. 134 (2006), no. 10, 3035-3040 (electronic), DOI 10.1090/S0002-9939-06-082499. MR2231629(2007d:44004)

[B] Sterling K. Berberian, Lectures in functional analysis and operator theory, Graduate Texts in Mathematics, No. 15, Springer-Verlag, New York, 1974. MR0417727 (54 \#5775)

[Bl] Grigoriy Blekherman, Positive Gorenstein ideals, Proc. Amer. Math. Soc. 143 (2015), no. 1, 69-86, DOI 10.1090/S0002-9939-2014-12253-2. MR3272733

[Co] John B. Conway, A course in functional analysis, 2nd ed., Graduate Texts in Mathematics, vol. 96, Springer-Verlag, New York, 1990. MR.1070713 (91e:46001)

[CF1] Raúl E. Curto and Lawrence A. Fialkow, Recursiveness, positivity, and truncated moment problems, Houston J. Math. 17 (1991), no. 4, 603-635. MR.1147276 (93a:47016)

[CF2] Raúl E. Curto and Lawrence A. Fialkow, Solution of the truncated complex moment problem for flat data, Mem. Amer. Math. Soc. 119 (1996), no. 568, x+52, DOI 10.1090/memo/0568. MR1303090 (96g:47009) 
[CF3] Raúl E. Curto and Lawrence A. Fialkow, Flat extensions of positive moment matrices: relations in analytic or conjugate terms, Nonselfadjoint operator algebras, operator theory, and related topics, Oper. Theory Adv. Appl., vol. 104, Birkhäuser, Basel, 1998, pp. 59-82. MR 1639649 (99i:47026)

[CF4] Raúl E. Curto and Lawrence A. Fialkow, Flat extensions of positive moment matrices: recursively generated relations, Mem. Amer. Math. Soc. 136 (1998), no. 648, x+56, DOI 10.1090/memo/0648. MR.1445490 (99d:47015)

[CF5] Raúl E. Curto and Lawrence A. Fialkow, Solution of the singular quartic moment problem, J. Operator Theory 48 (2002), no. 2, 315-354. MR1938799 (2003j:47017)

[CF6] Raúl E. Curto and Lawrence A. Fialkow, Solution of the truncated parabolic moment problem, Integral Equations Operator Theory 50 (2004), no. 2, 169-196, DOI 10.1007/s00020003-1275-3. MR2099788 (2005f:47041)

[CF7] Raúl E. Curto and Lawrence A. Fialkow, Truncated K-moment problems in several variables, J. Operator Theory 54 (2005), no. 1, 189-226. MR2168867 (2006e:47032)

[CF8] Raúl E. Curto and Lawrence A. Fialkow, Solution of the truncated hyperbolic moment problem, Integral Equations Operator Theory 52 (2005), no. 2, 181-218, DOI 10.1007/s00020-004-1340-6. MR.2216081 (2007a:47017)

[CF9] Raúl E. Curto and Lawrence A. Fialkow, An analogue of the Riesz-Haviland theorem for the truncated moment problem, J. Funct. Anal. 255 (2008), no. 10, 2709-2731, DOI 10.1016/j.jfa.2008.09.003. MR2464189 (2009i:47039)

[CF10] Raúl E. Curto and Lawrence A. Fialkow, Recursively determined representing measures for bivariate truncated moment sequences, J. Operator Theory 70 (2013), no. 2, 401-436, DOI 10.7900/jot.2011sep06.1943. MR3138363

[EF] Chirakkal Easwaran and Lawrence Fialkow, Positive linear functionals without representing measures, Oper. Matrices 5 (2011), no. 3, 425-434, DOI 10.7153/oam-05-30. MR2858497(2012m:47033)

[F] Lawrence A. Fialkow, Solution of the truncated moment problem with variety $y=x^{3}$, Trans. Amer. Math. Soc. 363 (2011), no. 6, 3133-3165, DOI 10.1090/S0002-9947-201105262-1. MR2775801 (2012c:47044)

[FN1] Lawrence Fialkow and Jiawang Nie, Positivity of Riesz functionals and solutions of quadratic and quartic moment problems, J. Funct. Anal. 258 (2010), no. 1, 328-356, DOI 10.1016/j.jfa.2009.09.015. MR2557966 (2010j:47017)

[FN2] Lawrence Fialkow and Jiawang Nie, On the closure of positive flat moment matrices, J. Operator Theory 69 (2013), no. 1, 257-277, DOI 10.7900/jot.2010may11.1890. MR.3029497

[FN3] Lawrence Fialkow and Jiawang Nie, The truncated moment problem via homogenization and flat extensions, J. Funct. Anal. 263 (2012), no. 6, 1682-1700, DOI 10.1016/j.jfa.2012.06.004. MR2948227

[H] E. K. Haviland, On the momentum problem for distribution functions in more than one dimension. II, Amer. J. Math. 58 (1936), no. 1, 164-168, DOI 10.2307/2371063. MR.1507139

[KN] M. G. Krĕ̌n and A. A. Nudel'man, The Markov moment problem and extremal problems. Ideas and problems of P. L. Čebyšev and A. A. Markov and their further development; Translated from the Russian by D. Louvish; Translations of Mathematical Monographs, Vol. 50, American Mathematical Society, Providence, R.I., 1977. MR0458081(56 \#16284)

[HN] J. William Helton and Jiawang Nie, A semidefinite approach for truncated K-moment problems, Found. Comput. Math. 12 (2012), no. 6, 851-881, DOI 10.1007/s10208-0129132-x. MR2989475

[HeLa] Didier Henrion and Jean-Bernard Lasserre, GloptiPoly: global optimization over polynomials with Matlab and SeDuMi, ACM Trans. Math. Software 29 (2003), no. 2, 165-194, DOI 10.1145/779359.779363. MR2000881 (2004g:90084)

[Her] Domingo A. Herrero, Approximation of Hilbert space operators. Vol. 1, 2nd ed., Pitman Research Notes in Mathematics Series, vol. 224, Longman Scientific \& Technical, Harlow, 1989. MR 1088255 (91k:47002)

[Las] Jean B. Lasserre, Global optimization with polynomials and the problem of moments, SIAM J. Optim. 11 (2000/01), no. 3, 796-817, DOI 10.1137/S1052623400366802. MR:1814045 (2002b:90054)

[Lau] Monique Laurent, Sums of squares, moment matrices and optimization over polynomials, Emerging applications of algebraic geometry, IMA Vol. Math. Appl., vol. 149, 
Springer, New York, 2009, pp. 157-270, DOI 10.1007/978-0-387-09686-5_7. MR2500468 (2010j:13054)

[Rez] Bruce Reznick, Some concrete aspects of Hilbert's 17th Problem, Real algebraic geometry and ordered structures (Baton Rouge, LA, 1996), Contemp. Math., vol. 253, Amer. Math. Soc., Providence, RI, 2000, pp. 251-272, DOI 10.1090/conm/253/03936. MR 1747589 (2001i:11042)

[R] M. Riesz, Sur le problème des moments, Troisième Note, Arkiv für Matematik, Astronomi och Fysik 17 (1923), 1-52.

[SH] S. L. Salas and E. Hille, Calculus: One and several variables, Fourth Edition, John Wiley and Sons, 1982.

[S1] Konrad Schmüdgen, An example of a positive polynomial which is not a sum of squares of polynomials. A positive, but not strongly positive functional, Math. Nachr. 88 (1979), 385-390, DOI 10.1002/mana.19790880130. MR543417 (81b:12024)

[S2] Konrad Schmüdgen, The K-moment problem for compact semi-algebraic sets, Math. Ann. 289 (1991), no. 2, 203-206, DOI 10.1007/BF01446568. MR1092173(92b:44011)

[T] Vladimir Tchakaloff, Formules de cubatures mécaniques à coefficients non négatifs (French), Bull. Sci. Math. (2) 81 (1957), 123-134. MR0094632 (20 \#1145)

Department of Computer Science, State University of New York, New Paltz, New YORK 12561

E-mail address: fialkowl@newpaltz.edu 